T. Mabuchi

Nagoya Math. J.

Vol. 162 (2001), 41-63

\title{
VECTOR FIELD ENERGIES AND CRITICAL METRICS ON KÄHLER MANIFOLDS
}

\author{
TOSHIKI MABUCHI
}

\begin{abstract}
Associated with a Hamiltonian holomorphic vector field on a compact Kähler manifold, a nice functional on a space of Kähler metrics will be constructed as an integration of the bilinear pairing in [FM] contracted with the Hamiltonian holomorphic vector field. As applications, we have functionals $\hat{\mu}, \hat{\nu}$ whose critical points are extremal Kähler metrics or "Kähler-Einstein metrics" in the sense of [M4], respectively. Finally, the same method as used by [G1] allows us to obtain, from the convexity of $\hat{\nu}$, the uniqueness of "Kähler-Einstein metrics" on nonsingular toric Fano varieties possibly with nonvanishing Futaki character.
\end{abstract}

\section{$\S 1$. Introduction}

The purpose of this paper is to define, with applications to the study of critical metrics, some functional associated with a Hamiltonian holomorphic vector field (see the key observation stated below). Throughout this paper, we fix once and for all an $n$-dimensional compact complex connected manifold $M$ with a Kähler class $\kappa \in H^{1,1}(M, \mathbb{R})$. The Albanese map of $M$ to the Albanese variety $\operatorname{Alb}(M)$ induces a complex Lie group homomorphism

$$
a_{M}: \operatorname{Aut}^{0}(M) \longrightarrow \operatorname{Aut}^{0}(\operatorname{Alb}(M))(\cong \operatorname{Alb}(M))
$$

between the identity components of the groups of holomorphic automorphisms of $M$ and $\operatorname{Alb}(M)$. Then the identity component $G:=\operatorname{Ker}^{0} a_{M}$ of the kernel of $a_{M}$ is a linear algebraic group (see [Fj]). Let $\mathcal{K}$ be the set of all Kähler metrics on $M$ in the Kähler class $\kappa$, where a Kähler metric and the associated Kähler form are used interchangeably. For each $\omega \in \mathcal{K}$, we write $\omega$ as

$$
\omega=\sqrt{-1} \sum_{\alpha, \beta} g_{\alpha \bar{\beta}} d z^{\alpha} \wedge d z^{\bar{\beta}}
$$

Received August 27, 1999.

Revised January 6, 2000.

1991 Mathematics Subject Classification: Primary 53C55; secondary 14J45; 14J50; $32 \mathrm{~J} 25$. 
in terms of a system $\left(z^{1}, z^{2}, \ldots, z^{n}\right)$ of holomorphic local coordinates on $M$. Put $A_{\kappa}:=\int_{M} \omega^{n}=\kappa^{n}[M]$. To each complex-valued smooth function $\varphi$ on $X$, we associate a complex vector field $\operatorname{grad}_{\omega}^{\mathbb{C}} \varphi$ on $M$ of type $(1,0)$ by

$$
\operatorname{grad}_{\omega}^{\mathbb{C}} \varphi:=\frac{1}{\sqrt{-1}} \sum_{\alpha, \beta} g^{\bar{\beta} \alpha} \frac{\partial \varphi}{\partial z^{\bar{\beta}}} \frac{\partial}{\partial z^{\alpha}} .
$$

Consider the complex Lie subalgebra $\mathfrak{g}$ of $H^{0}(M, \mathcal{O}(T M))$ corresponding to the complex Lie subgroup $G$ of $\operatorname{Aut}^{0}(M)$. Let $\tilde{\mathfrak{g}}_{\omega}$ be the space of all complex smooth functions $\varphi \in C^{\infty}(M)_{\mathbb{C}}$ on $M$ such that $\operatorname{grad}_{\omega}^{\mathbb{C}} \varphi$ is a holomorphic vector field on $M$ and that $\int_{M} \varphi \omega^{n} / A_{\kappa}=0$. Then we have an isomorphism of complex Lie algebras

$$
\iota_{\omega}: \tilde{\mathfrak{g}}_{\omega} \cong \mathfrak{g}, \quad \varphi \longleftrightarrow \iota_{\omega}(\varphi):=\operatorname{grad}_{\omega}^{\mathbb{C}} \varphi,
$$

where $\tilde{\mathfrak{g}}_{\omega}$ has a natural structure of a complex Lie algebra in terms of the Poisson bracket by $\omega$. Put $\tilde{\mathfrak{k}}_{\omega}:=\left\{\varphi \in \tilde{\mathfrak{g}}_{\omega} ; \varphi\right.$ is real-valued on $\left.M\right\}$ and $\mathfrak{k}_{\omega}:=$ $\iota_{\omega}\left(\tilde{\mathfrak{k}}_{\omega}\right)$. Then the real Lie subgroup $K_{\omega}$ of $G$ generated by the Lie subalgebra $\mathfrak{k}_{\omega}$ of $\mathfrak{g}$ is nothing but the identity component of the group of the isometries in $G$ of the compact Kähler manifold $(M, \omega)$. Put $\mathcal{K}_{V}:=\left\{\omega \in \mathcal{K} ; V \in \mathfrak{k}_{\omega}\right\}$, $V \in \mathfrak{g}$. Fix an element $\omega$ in $\mathcal{K}_{V}$ by assuming $\mathcal{K}_{V} \neq \emptyset$. Put

$$
\omega_{\psi}:=\omega+\sqrt{-1} \partial \bar{\partial} \psi, \quad \psi \in C^{\infty}(M)_{\mathbb{R}} .
$$

By sending $\psi$ to $\omega_{\psi}$, we have a surjection of $\tilde{\mathcal{K}}_{V}:=\left\{\psi \in \tilde{\mathcal{K}} ; \omega_{\psi} \in \mathcal{K}_{V}\right\}$ onto $\mathcal{K}_{V}$, where $\tilde{\mathcal{K}}$ denotes the set of all $\psi \in C^{\infty}(M)_{\mathbb{R}}$ such that $\omega_{\psi} \in \mathcal{K}$. Given a one-parameter family $\psi_{t} \in \tilde{\mathcal{K}}_{V}, a \leq t \leq b$, we say that $\left\{\psi_{t} ; a \leq t \leq b\right\}$ is a smooth path in $\tilde{\mathcal{K}}_{V}$, if the map of $M \times[a, b]$ to $\mathbb{R}$ sending $(x, t)$ to $\psi_{t}(x)$ is $C^{\infty}$. For such a smooth path $\left\{\psi_{t} ; a \leq t \leq b\right\}$, we put $\dot{\psi}_{t}:=(\partial / \partial t)\left(\psi_{t}\right)$ for simplicity. A key observation is ${ }^{1}$

Proposition A. Let $V$ be a holomorphic vector field belonging to $\mathfrak{g}$ such that $\mathcal{K}_{V} \neq \emptyset$. Then there exists a functional $\eta_{V}: \mathcal{K}_{V} \rightarrow \mathbb{R}$ satisfying the equality

$$
\frac{d}{d t} \eta_{V}\left(\omega_{t}\right)=\int_{M} \varphi_{t} \dot{\psi}_{t} \omega_{t}^{n} / A_{\kappa}, \quad a \leq t \leq b,
$$

for every smooth path $\left\{\psi_{t} ; a \leq t \leq b\right\}$ in $\tilde{\mathcal{K}}_{V}$, where we set $\omega_{t}:=\omega_{\psi_{t}}$, and the functions $\varphi_{t} \in \tilde{\mathfrak{k}}_{\omega_{t}}, a \leq t \leq b$, on $M$ are such that $V=\operatorname{grad}_{\omega_{t}}^{\mathbb{C}} \varphi_{t}$.

\footnotetext{
${ }^{1} \mathrm{My}$ sincere gratitude is due to Prof. Ryoichi Kobayashi who invited me to present this key observation in a lecture at Nagoya University in 1997. Arguments as in the proof of this were also used independently by [GC].
} 
For $W_{1}, W_{2} \in \mathfrak{g}$, we put $\left(W_{1}, W_{2}\right)_{\omega}:=\int_{M} \iota_{\omega}^{-1}\left(W_{1}\right) \iota_{\omega}^{-1}\left(W_{2}\right) \omega^{n} / A_{\kappa}$, which is independent of the choice of $\omega$ in $\mathcal{K}$, and will be denoted also by $\left(W_{1}, W_{2}\right)_{\kappa}($ cf. $[\mathrm{FM}])$. Such independence plays a crucial role in [FM], and Proposition A above gives some explanation for this independence (see (3.3)). Moreover, for $V$ as above, $\eta_{V}$ satisfies (cf. $\S 3$ )

$$
\frac{d}{d t}\left\{\eta_{V}\left(g_{t}^{*} \omega^{\prime}\right)\right\}=2 \operatorname{Im}(V, W)_{\mathcal{K}}, \quad \text { for all } W \in \mathfrak{z}(V) \text { and } \omega^{\prime} \in \mathcal{K}_{V}
$$

where $\mathfrak{z}(V)$ is the centralizer $\{W \in \mathfrak{g} ;[W, V]=0\}$ of $V$ in $\mathfrak{g}$, and for any $z \in \mathbb{C}, \operatorname{Re} z$ and $\sqrt{-1} \operatorname{Im} z$ denote the real part and the imaginary part of $z=\operatorname{Re} z+\sqrt{-1} \operatorname{Im} z$, respectively. Let $\underline{\mathcal{K}}$ denote the nonempty subset of $\mathcal{K}$ consisting of all $\omega \in \mathcal{K}$ such that $K_{\omega}$ is maximal compact in $G$. Then Proposition A allows us to construct functionals, $\hat{\mu}_{V}: \mathcal{K}_{V} \rightarrow \mathbb{R}, \hat{\mu}: \underline{\mathcal{K}} \rightarrow \mathbb{R}$ and $\hat{\nu}: \underline{\mathcal{K}} \rightarrow \mathbb{R}$, such that ${ }^{2}$

(1) all critical points for $\hat{\mu}_{V}$ and $\hat{\mu}$ are both extremal Kähler metrics;

(2) the set of the critical points for $\hat{\nu}$ consists all "Kähler-Einstein metrics" on $M$,

where for the functional $\hat{\mu}$, the pair $(M, \kappa)$ is assumed to be quantized (cf. $\S 5$ ), and for the functional $\hat{\nu}$, the cohomology class $\kappa$ is assumed to be $2 \pi c_{1}(M)_{\mathbb{R}}$. Note also that, in (2) above, $M$ possibly has nonvanishing Futaki character, where the terminology "Kähler-Einstein metric" is used in the sense of [M4]. We also have (see Propositions 5.7 and 6.5 and nearby arguments):

\section{ThEOREM B. The functionals $\hat{\mu}$ and $\hat{\nu}$ are $G$-invariant.}

From moduli-theoretic points of view, this $G$-invariance would be one of the most important properties featuring the functionals $\hat{\mu}$ and $\hat{\nu}$ above. By the convexity of $\hat{\nu}$, the method used by Guan in [G1] for extremal Kähler metrics now implies

Theorem C. (see [M5] for a more general case) Let $M$ be a nonsingular toric Fano variety, defined over $\mathbb{C}$, possibly with nonvanishing Futaki character. Then "Kähler-Einstein metrics" (cf. [M4]) on $M$ in the class $2 \pi c_{1}(M)_{\mathbb{R}}$ is unique, if any, up to the action of $G=\operatorname{Aut}^{0}(M)$.

\footnotetext{
${ }^{2}$ An important point is that both $\hat{\mu}$ and $\hat{\nu}$ are defined "globally" on $\underline{\mathcal{K}}$ without specifying any maximal compact subgroup of $G$. Such a condition of globality has never been studied seriously by any other authors.
} 


\section{$\S$ 2. Proof of Proposition A}

For each $V \in \mathfrak{g}$, let $V_{\mathbb{R}}$ denote the real vector field $V+\bar{V}$ on $M$ corresponding to the holomorphic vector field $V$ on $M$. Then the one-parameter group $\exp \left(t V_{\mathbb{R}}\right), t \in \mathbb{R}$, on $M$ generated by the vector field $V_{\mathbb{R}}$ comes from the action on $M$ of the one-parameter group $\exp t V, t \in \mathbb{R}$, in $G$. Hence, if there is no fear of confusion, we use $\exp t V$ and $\exp \left(t V_{\mathbb{R}}\right)$ interchangeably. Assuming $\mathcal{K}_{V} \neq \emptyset$, let $\omega \in \mathcal{K}_{V}$. Then the one-parameter group $P_{V}:=\left\{\exp \left(t V_{\mathbb{R}}\right) ; t \in \mathbb{R}\right\}$ has a compact closure $\bar{P}_{V}$ in $G$, since $\bar{P}_{V}$ is closed in the compact group $K_{\omega}$. Therefore

$$
\tilde{\mathcal{K}}_{V}=\left\{\psi \in \tilde{\mathcal{K}} ; V_{\mathbb{R}} \psi=0\right\}=\left\{\psi \in \tilde{\mathcal{K}} ; \psi \text { is } \bar{P}_{V} \text {-invariant }\right\} .
$$

For $\omega$ as above, let $\sigma(\omega)$ and $\square_{\omega}$ be respectively the corresponding scalar curvature and the Laplacian on functions defined by

$$
\sigma(\omega):=\sum_{\alpha, \beta} g^{\bar{\beta} \alpha} R_{\alpha \bar{\beta}}, \quad \square_{\omega}=\sum_{\alpha, \beta} g^{\bar{\beta} \alpha} \frac{\partial^{2}}{\partial z^{\alpha} \partial z^{\bar{\beta}}},
$$

where $\sum_{\alpha, \beta} R_{\alpha \bar{\beta}} d z^{\alpha} \wedge d z^{\bar{\beta}}$ denotes the Ricci form $R(\omega):=\sqrt{-1} \bar{\partial} \partial \log \omega^{n}$ for $\omega$. For each $\omega_{\psi} \in \mathcal{K}_{V}$, its scalar curvature $\sigma\left(\omega_{\psi}\right)$ and Laplacian $\square_{\omega_{\psi}}$ are denoted sometimes by $\sigma(\psi)$ and $\square_{\psi}$ respectively. To each pair $\left(\psi_{1}, \psi_{2}\right) \in$ $\tilde{\mathcal{K}}_{V} \times \tilde{\mathcal{K}}_{V}$, we associate $E_{V}\left(\psi^{\prime}, \psi^{\prime \prime}\right) \in \mathbb{R}$ by

$$
E_{V}\left(\psi^{\prime}, \psi^{\prime \prime}\right):=\int_{a}^{b}\left(\int_{M} \varphi_{t} \dot{\psi}_{t} \omega_{\psi_{t}}^{n} / A_{\kappa}\right) d t
$$

where $\left\{\psi_{t} ; a \leq t \leq b\right\}$ is an arbitrary piecewise smooth path in $\tilde{\mathcal{K}}_{V}$ satisfying $\psi_{a}=\psi^{\prime}$ and $\psi_{b}=\psi^{\prime \prime}$, and the functions $\varphi_{t} \in \tilde{\mathfrak{k}}_{\omega_{t}}, a \leq t \leq b$, on $M$ are such that

$$
V=\operatorname{grad}_{\omega_{t}}^{\mathbb{C}} \varphi_{t}
$$

with $\omega_{t}:=\omega_{\psi_{t}}$. Now by setting $\eta_{V}\left(\omega_{\psi}\right):=E_{V}(0, \psi)$, we can easily reduce the proof of Proposition A to showing the following theorem:

TheOREM 2.3. $E_{V}\left(\psi^{\prime}, \psi^{\prime \prime}\right)$ above is independent of the choice of the path $\left\{\psi_{t} ; a \leq t \leq b\right\}$, in $\tilde{\mathcal{K}}_{V}$, and therefore well-defined. In particular,

$$
E_{V}\left(\psi, \psi^{\prime}\right)+E_{V}\left(\psi^{\prime}, \psi^{\prime \prime}\right)+E_{V}\left(\psi^{\prime \prime}, \psi\right)=0 \text { for all } \psi, \psi^{\prime}, \psi^{\prime \prime} \in \tilde{\mathcal{K}}_{V}
$$

$$
E_{V}(\psi, \psi+C)=0 \text { for all } \psi \in \tilde{\mathcal{K}}_{V} \text { and all } C \in \mathbb{R} \text {. }
$$


In view of the assumption $\omega \in \mathcal{K}_{V}$, we have $V=\operatorname{grad}_{\omega}^{\mathbb{C}} \phi$ for $\phi:=$ $\iota_{\omega}^{-1}(V) \in \tilde{\mathfrak{k}}_{\omega}$. Then the following lemma is essential in the proof of Theorem 2.3:

LEMMA 2.6. (cf. [FM;p.208]) The equality $\varphi_{t}=\phi+\sqrt{-1} V \psi_{t}$ holds for all $a \leq t \leq b$.

By using this lemma, we shall now prove Theorem 2.3.

Proof of Theorem 2.3. Define a map $\Psi=\Psi(s, t)$ of the rectangle $R:=$ $[0,1] \times[a, b]$ to $\tilde{\mathcal{K}}_{V}$ by $\Psi(s, t):=s \psi_{t}$ for $(s, t) \in[0,1] \times[a, b]$. Since $\left\{\psi_{t} ; a \leq\right.$ $t \leq b\}$ is piecewise smooth, there exists a partition $a=a_{0}<a_{1}<a_{2}<$ $\ldots<a_{r}=b$ of the interval $[a, b]$ such that $\left\{\psi_{t} ; a_{i-1} \leq t \leq a_{i}\right\}$ is smooth for each $i \in\{1,2, \ldots, r\}$. We then divide the proof of Theorem 2.3 into the following two steps:

Step 1: For simplicity, put $\omega_{s, t}:=\omega_{\Psi(s, t)}$ for each $(s, t) \in R$. Then by Lemma 2.6, we have $V=\operatorname{grad}_{\omega_{s, t}}^{\mathbb{C}} \Phi(s, t)$, where $\Phi=\Phi(s, t)$ is defined by $\Phi(s, t):=\phi+\sqrt{-1} V \Psi(s, t) \in \tilde{\mathfrak{k}}_{\omega_{s, t}}$. Here, $\Phi(1, t)=\phi+\sqrt{-1} V \psi_{t}=\varphi_{t}$. The purpose of this step is to show that

$$
\int_{a_{i-1}}^{a_{i}}\left(\int_{M} \varphi_{t} \dot{\psi}_{t} \omega_{\Psi}^{n} / A_{\kappa}\right) d t=\left.\int_{0}^{1}\left(\int_{M} \Phi \frac{\partial \Psi}{\partial s} \omega_{\Psi}^{n} / A_{\kappa}\right) d s\right|_{t=a_{i-1}} ^{t=a_{i}}
$$

Let $\Theta:=\left(\int_{M} \Phi \Psi_{s} \omega_{\Psi}^{n} / A_{\kappa}\right) d s+\left(\int_{M} \Phi \Psi_{t} \omega_{\Psi}^{n} / A_{\kappa}\right) d t$, where $\Psi_{s}:=\partial \Psi / \partial s$ and $\Psi_{t}:=\partial \Psi / \partial t$. Moreover, we put $\Phi_{s}:=\partial \Phi / \partial s$ and $\Phi_{t}:=\partial \Phi / \partial t$. For a suitable orientation of the rectangle $R$, its boundary $\partial R$ is written as a sum $\gamma_{1}+\gamma_{2}-\gamma_{3}-\gamma_{4}$, where

$$
\begin{array}{ll}
\gamma_{1}:=\left\{\left(s, a_{i-1}\right) ; 0 \leq s \leq 1\right\}, & \gamma_{2}:=\left\{(1, t) ; a_{i-1} \leq t \leq a_{i}\right\}, \\
\gamma_{3}:=\left\{\left(s, a_{i}\right) ; 0 \leq s \leq 1\right\}, & \gamma_{4}:=\left\{(0, t) ; a_{i-1} \leq t \leq a_{i}\right\} .
\end{array}
$$

Then by the Stokes theorem, $\int_{R} d \Theta=\int_{\partial R} \Theta=\int_{\gamma_{1}+\gamma_{2}-\gamma_{3}-\gamma_{4}} \Theta$. Moreover, the pullback of $\Theta$ to $\gamma_{4}$ vanishes. Hence, $\int_{R} d \Theta$ is just

$$
\begin{array}{r}
-\int_{\gamma_{3}-\gamma_{1}} \Theta+\int_{\gamma_{2}} \Theta=-\left.\int_{0}^{1}\left(\int_{M} \Phi \Psi_{s} \omega_{\Psi}^{n} / A_{\kappa}\right) d s\right|_{t=a_{i-1}} ^{t=a_{i}} \\
+\int_{a_{i-1}}^{a_{i}}\left(\int_{M} \varphi_{t} \dot{\psi}_{t} \omega_{\Psi}^{n} / A_{\kappa}\right) d t
\end{array}
$$


Thus the proof of (2.7) is reduced to showing the vanishing $d \Theta=0$ on the rectangle $R$. In terms of a system of holomorphic local coordinates $\left(z^{1}, z^{2}, \ldots, z^{n}\right)$, we write the Kähler metric $\omega_{\Psi}=\omega_{\Psi(s, t)}=\omega_{s, t}$ in the form

$$
\omega_{\Psi}=\sqrt{-1} \sum_{\alpha, \beta} g_{\Psi_{\alpha} \bar{\beta}} d z^{\alpha} \wedge d z^{\bar{\beta}} .
$$

Then for $\zeta_{1}, \zeta_{2} \in C^{\infty}(M)_{\mathbb{C}}$, we can define the Poisson bracket $\left[\zeta_{1}, \zeta_{2}\right]_{\Psi}$ of $\zeta_{1}$ and $\zeta_{2}$ relative to the Kähler metric $\omega_{\Psi}$ by

$$
\left[\zeta_{1}, \zeta_{2}\right]_{\Psi}:=\sqrt{-1} \sum_{\alpha, \beta} g_{\Psi}^{\bar{\beta} \alpha}\left(\frac{\partial \zeta_{1}}{\partial z^{\alpha}} \frac{\partial \zeta_{2}}{\partial z^{\bar{\beta}}}-\frac{\partial \zeta_{1}}{\partial z^{\bar{\beta}}} \frac{\partial \zeta_{2}}{\partial z^{\alpha}}\right)
$$

Let $(,)_{\Psi}: A^{q}(M)_{\mathbb{C}} \times A^{q}(M)_{\mathbb{C}} \rightarrow C^{\infty}(M)_{\mathbb{C}}$ be the pointwise Hermitian pairing associated with the Kähler metric $\omega_{\Psi}$, where $A^{q}(M)_{\mathbb{C}}$ denotes the space of all complex-valued smooth $q$-forms on $M$. By a straightforward computation,

$$
\begin{aligned}
d \Theta=d s & \wedge d t \int_{M}\left\{\frac{\partial}{\partial s}\left(\Phi \Psi_{t} \omega_{\Psi}^{n} / A_{\kappa}\right)-\frac{\partial}{\partial t}\left(\Phi \Psi_{s} \omega_{\Psi}^{n} / A_{\kappa}\right)\right\} \\
=d s & \wedge d t \int_{M}\left\{\left(\Phi_{s} \Psi_{t}-\Phi_{t} \Psi_{s}\right)+\Phi \Psi_{t}\left(\square_{\Psi} \Psi_{s}\right)-\Phi \Psi_{s}\left(\square_{\Psi} \Psi_{t}\right)\right\} \omega_{\Psi}^{n} / A_{\kappa} \\
= & \sqrt{-1} d s \wedge d t \int_{M}\left\{\Psi_{t}\left(V \Psi_{s}\right)-\Psi_{s}\left(V \Psi_{t}\right)\right\} \omega_{\Psi}^{n} / A_{\kappa} \\
& +d s \wedge d t \int_{M}\left\{-\left(\bar{\partial}\left(\Phi \Psi_{t}\right), \bar{\partial} \Psi_{s}\right)_{\Psi}+\left(\bar{\partial}\left(\Phi \Psi_{s}\right), \bar{\partial} \Psi_{t}\right)_{\Psi}\right\} \omega_{\Psi}^{n} / A_{\kappa} .
\end{aligned}
$$

On the other hand, by $V=\operatorname{grad}_{\omega}^{\mathbb{C}} \Phi$, we obtain

$$
\begin{aligned}
& -\left(\bar{\partial}\left(\Phi \Psi_{t}\right), \bar{\partial} \Psi_{s}\right)_{\Psi}+\left(\bar{\partial}\left(\Phi \Psi_{s}\right), \bar{\partial} \Psi_{t}\right)_{\Psi} \\
= & \sqrt{-1} \Phi\left[\Psi_{s}, \Psi_{t}\right]_{\Psi}-\left(\Psi_{t} \bar{\partial} \Phi, \bar{\partial} \Psi_{s}\right)_{\Psi}+\left(\Psi_{s} \bar{\partial} \Phi, \bar{\partial} \Psi_{t}\right)_{\Psi} \\
= & \sqrt{-1}\left\{\Phi\left[\Psi_{s}, \Psi_{t}\right]_{\Psi}-\Psi_{t}\left(V \Psi_{s}\right)+\Psi_{s}\left(V \Psi_{t}\right)\right\} .
\end{aligned}
$$

These together with $V_{\mathbb{R}} \psi_{t}=0$ (see (2.1)) show the vanishing of $d \Theta$ as follows:

$$
\begin{aligned}
d \Theta & =\sqrt{-1} d s \wedge d t \int_{M} \Phi\left[\Psi_{s}, \Psi_{t}\right]_{\Psi} \omega_{\Psi}^{n} / A_{\kappa} \\
& =\sqrt{-1} d s \wedge d t \int_{M}\left[\Phi, \Psi_{s}\right]_{\Psi} \Psi_{t} \omega_{\Psi}^{n} / A_{\kappa}
\end{aligned}
$$




$$
\begin{aligned}
& =\sqrt{-1} d s \wedge d t \int_{M}\left(V_{\mathbb{R}} \Psi_{s}\right) \Psi_{t} \omega_{\Psi}^{n} / A_{\kappa} \\
& =\sqrt{-1} d s \wedge d t \int_{M}\left(V_{\mathbb{R}} \psi_{t}\right) \Psi_{t} \omega_{\Psi}^{n} / A_{\kappa}=0 .
\end{aligned}
$$

Step 2: Consider the equality $(2.7)$ for $i=1,2, \ldots, r$. By adding them up, we obtain

$$
\int_{a}^{b}\left(\int_{M} \varphi_{t} \dot{\psi}_{t} \omega_{\psi_{t}}^{n} / A_{\kappa}\right) d t=\left.\int_{0}^{1}\left(\int_{M} \Phi \psi_{t} \omega_{\Psi}^{n} / A_{\kappa}\right) d s\right|_{t=a} ^{t=b}
$$

Therefore, the left-hand side is independent of the choice of the piecewise smooth path $\left\{\psi_{t} ; a \leq t \leq b\right\}$ in $\tilde{\mathcal{K}}_{V}$, as long as $\psi_{a}=\psi^{\prime}$ and $\psi_{b}=\psi^{\prime \prime}$. Then (2.4) is now immediate. For $(2.5)$, let $\psi_{t}:=\psi+t C$, where $t \in[0,1]$. Put $\omega_{t}:=\omega_{\psi_{t}}$ for simplicity. For each $t$, consider the associated $\varphi_{t} \in \tilde{\mathfrak{k}}_{\omega_{t}}$ satisfying $V=\operatorname{grad}_{\omega_{t}}^{\mathbb{C}} \varphi_{t}$. Then,

$$
E(\psi, \psi+C)=\int_{0}^{1} \int_{M} \varphi_{t} \dot{\psi}_{t} \omega_{\psi_{t}}^{n} / A_{\kappa}=C \int_{0}^{1}\left(\int_{M} \varphi_{t} \omega_{t}^{n} / A_{\kappa}\right)=0
$$

\section{$\S$ 3. An application to the study of the bilinear pairing $(,)_{\kappa}$ on $\mathfrak{k}^{\mathbb{C}}$}

Let $V \in \mathfrak{g}$ be such that $\omega \in \mathcal{K}_{V} \neq \emptyset$. We put $V^{g}:=\left(g^{-1}\right)_{*} V=$ $\operatorname{Ad}\left(g^{-1}\right) V$ for all $g \in G$. Let $\omega_{0}$ and $\omega_{1}$ be arbitrary elements in $\mathcal{K}_{V}$. We choose a smooth path $\left\{\psi_{t} \in \tilde{\mathcal{K}}_{V} ; a \leq t \leq b\right\}$ in $\tilde{\mathcal{K}}_{V}$ such that the corresponding path $\omega_{t}:=\omega_{\psi_{t}}, a \leq t \leq b$, connecting $\omega_{0}$ and $\omega_{1}$ in $\mathcal{K}_{V}$ satisfies

$$
\int_{M} \dot{\psi}_{t} \omega_{t}^{n} / A_{\kappa}=0 \quad \text { for all } t
$$

For each $t$, we can write $V=\operatorname{grad}_{\omega_{t}}^{\mathbb{C}} \varphi_{t}$ for some unique $\varphi_{t} \in \tilde{\mathfrak{k}}_{\omega_{t}}$. On the other hand, for every $g \in G$, we see that $g^{*} \omega_{0}, g^{*} \omega_{1} \in \mathcal{K}_{V^{g}}$, because the condition $V \in \mathfrak{k}_{\omega}$ always implies $V^{g} \in \mathfrak{k}_{g^{*} \omega}$. Now, $g^{*} \omega_{t}=g^{*} \omega+\sqrt{-1} \partial \bar{\partial}\left(g^{*} \psi_{t}\right)$, $a \leq t \leq b$, is a path in $\mathcal{K}_{V^{g}}$ connecting the metrics $g^{*} \omega_{0}, g^{*} \omega_{1}$ and satisfying $\int_{M}\left(g^{*} \dot{\psi}_{t}\right) g^{*} \omega_{t}^{n} / A_{\kappa}=0$ for all $t$. In view of $V^{g}=\operatorname{grad}_{g^{*} \omega_{t}}^{\mathbb{C}} g^{*} \varphi_{t} \in \tilde{\mathfrak{k}}_{g^{*} \omega_{t}}$, we see that

$$
\begin{aligned}
E_{V^{g}}\left(g^{*} \omega_{0}, g^{*} \omega_{1}\right) & =\int_{a}^{b}\left(\int_{M} g^{*} \varphi_{t} g^{*} \dot{\psi}_{t} g^{*} \omega_{t}^{n} / A_{\kappa}\right) d t \\
& =\int_{a}^{b}\left(\int_{M} \varphi_{t} \dot{\psi}_{t} \omega_{t}^{n} / A_{\kappa}\right) d t=E_{V}\left(\omega_{0}, \omega_{1}\right)
\end{aligned}
$$


Consider the algebraic subgroup $Z(V):=\left\{g \in G ; V^{g}=V\right\}$ of $G$. Obviously, $Z(V)$ has the Lie algebra $\mathfrak{z}(V)$. We now claim that

Lemma 3.2. $E_{V}\left(\omega_{0}, g^{*} \omega_{0}\right)=E_{V}\left(\omega_{1}, g^{*} \omega_{1}\right)$ for all $g \in Z(V)$ and $\omega_{1}$, $\omega_{2} \in \mathcal{K}_{V}$.

Proof. By $g \in Z(V)$, we have $V^{g}=V$. Hence by $(3.1), E_{V}\left(g^{*} \omega_{0}, g^{*} \omega_{1}\right)$ $=E_{V}\left(\omega_{0}, \omega_{1}\right)=E_{V}\left(\omega_{0}, g^{*} \omega_{0}\right)+E_{V}\left(g^{*} \omega_{0}, g^{*} \omega_{1}\right)-E_{V}\left(\omega_{1}, g^{*} \omega_{1}\right)$. Then the required equality $E_{V}\left(\omega_{0}, g^{*} \omega_{0}\right)=E_{V}\left(\omega_{1}, g^{*} \omega_{1}\right)$ follows immediately.

For a maximal compact subgroup $K$ of $G$, let $\omega_{0}, \omega_{1} \in \mathcal{K}^{K}$, where $\mathcal{K}^{K}$ denotes the set of all $K$-invariant elements in $\mathcal{K}$. Let $\mathfrak{k}$ denote the Lie subalgebra of $\mathfrak{g}$ corresponding to the Lie subgroup $K$ of $G$. Then $\mathfrak{k}_{\omega_{0}}=$ $\mathfrak{k}_{\omega_{1}}=\mathfrak{k}$. Let $V, W \in \mathfrak{t}$, where $\mathfrak{t}$ is a maximal toral subalgebra of $\mathfrak{k}$. We first observe that $\omega_{0}, \omega_{1} \in \mathcal{K}_{V}$. Moreover, we can write

$$
V=\operatorname{grad}_{\omega_{i}}^{\mathbb{C}} v_{i} \quad \text { and } \quad W=\operatorname{grad}_{\omega_{i}}^{\mathbb{C}} w_{i}, \quad i=0,1
$$

for some $v_{i}, w_{i} \in \tilde{\mathfrak{k}}_{\omega_{i}}$. Put $g_{t}:=\exp (t \sqrt{-1} W)=\exp \left\{t(\sqrt{-1} W)_{\mathbb{R}}\right\}$. This $g_{t}$ belongs to $Z(V)$ for all $t \in \mathbb{R}$. Write $g_{t}^{*} \omega_{i}=\omega_{i}+\sqrt{-1} \partial \bar{\partial} \psi_{i, t}$ for some smooth one-parameter families $\left\{\psi_{i, t} ; t \in \mathbb{R}\right\}$ of real-valued $C^{\infty}$ functions on $M$. Note that

$$
\left(\dot{\psi}_{i, t}\right)_{\mid t=0}=2 w_{i}+C_{i}, \quad i=0,1
$$

for some constants $C_{i} \in \mathbb{R}$. Now by Lemma $3.2, E_{V}\left(\omega_{0}, g_{t}^{*} \omega_{0}\right)=$ $E_{V}\left(\omega_{1}, g_{t}^{*} \omega_{1}\right)$ for all $t$. Differentiating this with repect to $t$ at $t=0$, we obtain

$$
\int_{M} v_{0} w_{0} \omega_{0}^{n} / A_{\kappa}=\int_{M} v_{1} w_{1} \omega_{1}^{n} / A_{\kappa} .
$$

Recall that the identity (3.3) is the key point in proving the well-definition of the bilinear pairing $\mathfrak{k}^{\mathbb{C}} \times \mathfrak{k}^{\mathbb{C}} \ni(V, W) \mapsto(V, W)_{\kappa} \in \mathbb{C}$ (cf. $\left.[\mathrm{FM}] ; \S 1\right)$, where $\mathfrak{k}^{\mathbb{C}}$ denotes the complexification of $\mathfrak{k}$ in $\mathfrak{g}$.

Remark. Let $W \in \mathfrak{z}(V)$ and $V \in \mathfrak{g}$ with $\mathcal{K}_{V} \neq \emptyset$. Let $\omega$ be an arbitrary element of $\mathcal{K}_{V}$. Put $g_{t}:=\exp t W_{\mathbb{R}}$ and $\omega_{t}:=g_{t}^{*} \omega, t \in \mathbb{R}$. Then we can write

$$
V=\operatorname{grad}_{\omega_{t}}^{\mathbb{C}} v_{t}, \quad \text { and } \quad W=\operatorname{grad}_{\omega_{t}}^{\mathbb{C}} w_{t}
$$

for some $v_{t} \in \tilde{\mathfrak{k}}_{\omega_{t}}$ and $w_{t} \in \tilde{\mathfrak{g}}_{\omega_{t}}$. Write $\omega_{t}=\omega+\sqrt{-1} \partial \bar{\partial} \psi_{t}$ for some smooth one-parameter family $\left\{\psi_{t} ; t \in \mathbb{R}\right\}$ of real-valued $C^{\infty}$ functions on $M$. Then

$$
\dot{\psi}_{t}=2 \operatorname{Im} w_{t}+C_{t}
$$


for some real constant $C_{t}$. Then by the definition of the functional $\eta_{V}$, we have the following equality (see also (1.2)), which is an important ingredient of the proof of $(3.3)$ :

$$
\frac{d}{d t} \eta_{V}\left(g_{t}^{*} \omega\right)=2 \operatorname{Im} \int_{M} v_{t} w_{t} \omega_{t}^{n} / A_{\kappa}=2 \operatorname{Im}(V, W)_{\kappa}, \quad t \in \mathbb{R} .
$$

Remark. Let $V \in \mathfrak{g}$ be such that $\omega \in \mathcal{K}_{V} \neq \emptyset$, and let $\mathbb{R}_{+}$denote the multiplicative group of all positive real numbers. Put $e_{V}(g):=$ $\exp \left(E_{V}\left(\omega, g^{*} \omega\right)\right)$. Then $e_{V}: Z(V) \rightarrow \mathbb{R}_{+}$defines a character of real Lie groups as follows:

$$
\begin{aligned}
& \log \left(e_{V}\left(g_{1} g_{2}\right)\right)=E_{V}\left(\omega,\left(g_{1} g_{2}\right)^{*} \omega\right)=E_{V}\left(\omega, g_{2}^{*} g_{1}^{*} \omega\right) \\
= & E_{V}\left(\omega, g_{1}^{*} \omega\right)+E_{V}\left(g_{1}^{*} \omega, g_{2}^{*} g_{1}^{*} \omega\right)=E_{V}\left(\omega, g_{1}^{*} \omega\right)+E_{V}\left(\omega, g_{2}^{*} \omega\right) \\
= & \log e_{V}\left(g_{1}\right)+\log e_{V}\left(g_{2}\right)
\end{aligned}
$$

i.e., $e_{V}\left(g_{1} g_{2}\right)=e_{V}\left(g_{1}\right) e_{V}\left(g_{2}\right)$ for all $g_{1}, g_{2} \in Z(V)$. Thus, $e_{V}: Z(V) \rightarrow \mathbb{R}_{+}$ is a group character of real Lie groups.

\section{$\S 4$. Functional $\hat{\mu}_{V}$ whose critical points are extremal Kähler metrics}

In this section, we fix an element $\omega$ in $\underline{\mathcal{K}}$. Then the group $K_{\omega}$ (see $\S 1$ ) is maximal compact in $G$. The extremal Kähler vector field $\mathcal{V}_{\omega} \in \mathfrak{k}_{\omega}$ (cf. $[\mathrm{FM}])$ is defined by

$$
\mathcal{V}_{\omega}=\operatorname{grad}_{\omega}^{\mathbb{C}}\left(\operatorname{pr}_{\omega} \sigma(\omega)\right)
$$

where $\operatorname{pr}_{\omega}: L^{2}(M, \omega)_{\mathbb{R}} \rightarrow \mathbb{R} \oplus \tilde{\mathfrak{k}}_{\omega}$ is the orthogonal projection from the space $L^{2}(M, \omega)_{\mathbb{R}}$ of all real-valued $L^{2}$-functions on the Kähler manifold $(M, \omega)$ onto its finite-dimensional subspace $\mathbb{R} \oplus \tilde{\mathfrak{k}}_{\omega}:=\left\{\varphi \in C^{\infty}(M)_{\mathbb{R}} ; \operatorname{grad}_{\omega}^{\mathbb{C}} \varphi \in \mathfrak{g}\right\}$. Then the orthogonal complement $\left(\mathbb{R} \oplus \tilde{\mathfrak{k}}_{\omega}\right)^{\perp}$ of $\mathbb{R} \oplus \tilde{\mathfrak{k}}_{\omega}$ in $L^{2}(M, \omega)_{\mathbb{R}}$ is exactly the kernel of $\operatorname{pr}_{\omega}$. In this section, we fix an element $\omega$ in $\underline{\mathcal{K}}$, and put

$$
V:=\mathcal{V}_{\omega}
$$

Then $\omega$ belongs to $\mathcal{K}_{V}$ obviously. Let $K_{\omega}^{\mathbb{C}}$ be the reductive algebraic subgroup of $G$ obtained as the complexification of $K_{\omega}$ in $G$. The corresponding Lie subalgebra of $\mathfrak{g}$ will be denoted by $\mathfrak{k}_{\omega}^{\mathbb{C}}$. Obviously, $V \in \mathfrak{k}_{\omega} \subset \mathfrak{k}_{\omega}^{\mathbb{C}} \subset \mathfrak{z}(V)$. We first observe that

LEMMA 4.1. $Z(V)$ is connected. 
Proof. By the Chevalley decomposition of $G$, we write $G$ as a semidirect product $K_{\omega}^{\mathbb{C}} \ltimes U$, where $U$ is the unipotent radical of $G$. Let $\mathfrak{u}$ be the Lie subalgebra of $\mathfrak{g}$ corresponding to $U$. Then every element of $Z(V)$ is written as $k(\exp W)$ for some $k \in K_{\omega}^{\mathbb{C}}$ and $W \in \mathfrak{u}$. By $K_{\omega}^{\mathbb{C}} \subset Z(V)$, we see that $\exp W \in Z(V)$, i.e., $V=\{\exp \operatorname{ad}(W)\} V$. Then Jordan's normal form of the linear map $\operatorname{ad}(W)$ of $\mathfrak{g}$ onto itself allows us to obtain $W \in \mathfrak{z}(V)$. Now, $k \exp (t W) \in Z(V)$ for all $0 \leq t \leq 1$. Thus, $Z(V)$ is connected.

We now put $\mathcal{H}_{V}:=\left\{\omega^{\prime} \in \underline{\mathcal{K}} ; \mathcal{V}_{\omega^{\prime}}=V\right\}$, where $\mathcal{V}_{\omega^{\prime}} \in \mathfrak{k}_{\omega^{\prime}}$ denotes the extremal Kähler vector field of $\omega^{\prime}$. Then $\mathcal{H}_{V}$ is a nonempty subset of $\underline{\mathcal{K}}$ satisfying

$$
\omega \in\left\{\omega^{\prime} \in \mathcal{K} ; \mathfrak{k}_{\omega^{\prime}}=\mathfrak{k}_{\omega}\right\} \subset \mathcal{H}_{V} \subset \mathcal{K}_{V} .
$$

Let $\tilde{\mathcal{H}}_{V}$ denote the set of all $\psi \in \tilde{\mathcal{K}}_{V}$ such that $\omega_{\psi} \in \mathcal{H}_{V}$. By a piecewise smooth path in $\tilde{\mathcal{H}}_{V}$, we mean a piecewise smooth path in $\tilde{\mathcal{K}}_{V}$ sitting in $\tilde{\mathcal{H}}_{V}$. For each $\psi \in \tilde{\mathcal{H}}_{V}$, we take an arbitrary piecewise smooth path $\left\{\psi_{t} ; a \leq t \leq\right.$ b\} in $\tilde{\mathcal{H}}_{V}$ such that $\psi_{a}=0$ and $\psi_{b}=\psi$. Then the restriction to $\mathcal{H}_{V}$ of the K-energy map $\mu: \mathcal{K} \rightarrow \mathbb{R}$ (cf. [M1]) is given by

$$
\mu\left(\omega_{\psi}\right):=-\int_{a}^{b}\left\{\int_{M}\left(\sigma\left(\omega_{t}\right)-C_{\kappa}\right) \dot{\psi}_{t} \omega_{t}^{n} / A_{\kappa}\right\} d t, \quad \psi \in \tilde{\mathcal{H}}_{V}
$$

where we put $\omega_{t}:=\omega_{\psi_{t}}$ for simplicity, and $C_{\kappa}$ is the real constant $\int_{M} \sigma(\omega) \omega^{n} / A_{\kappa}$. The set of the critical points for $\mu$ just consists of all Kähler metrics in $\mathcal{K}_{V}$ of constant scalar curvature. Define $\hat{\mu}_{V}: \mathcal{H}_{V} \rightarrow \mathbb{R}$ by

$$
\hat{\mu}_{V}:=\mu+\eta_{V}
$$

where $\eta_{V}$ is as in the introduction. For each $t$, we write $V=\operatorname{grad}_{\omega_{t}}^{\mathbb{C}} \varphi_{t}$ for some unique $\varphi_{t} \in \tilde{\mathfrak{k}}_{\omega_{t}}$. By $\operatorname{pr}_{\omega_{t}} \sigma\left(\omega_{t}\right)=C_{\kappa}+\varphi_{t}$, we see from the equalities (1.1), (4.2), (4.3) that

$$
\begin{aligned}
& \hat{\mu}_{V}\left(\omega_{\psi}\right) \\
& \quad=-\int_{a}^{b}\left(\int_{M}\left\{\sigma\left(\omega_{t}\right)-\operatorname{pr}_{\omega_{t}} \sigma\left(\omega_{t}\right)\right\} \dot{\psi}_{t} \omega_{t}^{n} / A_{\kappa}\right) d t, \quad \psi \in \tilde{\mathcal{H}}_{V},
\end{aligned}
$$

for $\left\{\psi_{t} ; a \leq t \leq b\right\}$ as above. Let $\omega^{\prime} \in \mathcal{H}_{V}$. Since $\sigma\left(\omega^{\prime}\right)-\operatorname{pr}_{\omega^{\prime}} \sigma\left(\omega^{\prime}\right)$ is a $K_{\omega^{\prime}}$-invariant function, $\omega^{\prime}$ can be perturbed in $\mathcal{H}_{V}$ to the form $\omega^{\prime}+$ $\sqrt{-1} \varepsilon \partial \bar{\partial}\left\{\sigma\left(\omega^{\prime}\right)-\operatorname{pr}_{\omega^{\prime}} \sigma\left(\omega^{\prime}\right)\right\}$, where $\varepsilon>0$ is sufficiently small. Since the equality $\sigma\left(\omega^{\prime}\right)=\operatorname{pr}_{\omega^{\prime}} \sigma\left(\omega^{\prime}\right)$ holds if and only if $\omega^{\prime}$ is an extremal Kähler metric, (4.4) above implies that 
Proposition 4.5. An element $\omega^{\prime}$ of $\mathcal{H}_{V}$ is a critical point for the functional $\hat{\mu}_{V}: \mathcal{H}_{V} \rightarrow \mathbb{R}$ if and only if $\omega^{\prime}$ is an extremal Kähler metric.

Remark. The functional $\hat{\mu}_{V}$ above was obtained by the author in 1994, though the result was unpublished. A little afterwards, Simanca (see [S1]) obtained a similar result. Guan [G1] studied such a functional independently and successfully, applying it to the uniqueness (modulo connected group actions) of extremal Kähler metrics in a Kähler class of a nonsingular toric variety.

\section{$\S 5$. Functional $\hat{\mu}: \underline{\mathcal{K}} \rightarrow \mathbb{R}$ for a quantized pair $(M, \kappa)$}

Throughout this section, we assume that the pair $(M, \kappa)$ is quantized, i.e., there exists a holomorphic line bundle $L$ over $M$ such that

(1) the Kähler class $\kappa$ in the introduction is $2 \pi c_{1}(L)_{\mathbb{R}}$;

(2) the $G$-action on $M$ lifts to a holomorphic $G$-action on $L$ preserving set-theoretically the image of the zero section of $L$.

For instance, if $M$ is a Fano manifold, then the pair $\left(M, c_{1}(M)_{\mathbb{R}}\right)$ is quantized by choosing the anticanonical bundle $K_{M}^{-1}$ as $L$. The main purpose of this section is to define a functional $\hat{\mu}: \underline{\mathcal{K}} \rightarrow \mathbb{R}$ for each quantized pair $(M, \kappa)$ from the functionals $\hat{\mu}_{V^{g}}: \mathcal{H}_{V^{g}} \rightarrow \mathbb{R}, g \in G$, (cf. §4) glued together.

Let $\mathfrak{u}$ be the Lie subalgebra of $\mathfrak{g}$ corresponding to the unipotent radical $U$ of $G$, where we write $G$ as a semi-direct product $K_{\omega}^{\mathbb{C}} \ltimes U$. Take a $\mathbb{C}$ basis $\left\{\mathcal{Y}_{1}, \mathcal{Y}_{2}, \ldots, \mathcal{Y}_{m}\right\}$ for $\mathfrak{u}$. Furthermore, let $\left\{\mathcal{X}_{1}, \mathcal{X}_{2}, \ldots, \mathcal{X}_{\ell}\right\}$ be an $\mathbb{R}$ basis for $\mathfrak{k}_{\omega}$, which is naturally regarded as a $\mathbb{C}$-basis for $\mathfrak{k}_{\omega}^{\mathbb{C}}$. We choose $1 \ll k \in \mathbb{Z}$ such that $L^{\otimes k}$ is very ample. Let $\left\{\sigma_{0}, \sigma_{1}, \ldots, \sigma_{r}\right\}$ be a $\mathbb{C}$-basis for $S:=H^{0}\left(M, L^{\otimes k}\right)$. Note that, via the $U$-action on $L$, the unipotent group $U$ acts naturally on $S$, which induces an infinitesimal action of $\mathfrak{u}$ on $S$. Since $U$ is unipotent, we may assume that

$$
\mathcal{Y}_{j} \sigma_{\lambda}= \begin{cases}0 & \text { if } 1 \leq j \leq m \text { and } \lambda=0 \\ \sum_{\mu=0}^{\lambda-1} b_{j, \lambda, \mu} \sigma_{\mu} & \text { if } 1 \leq j \leq m \text { and } 1 \leq \lambda \leq r\end{cases}
$$

for some complex numbers $b_{j, \lambda, \mu} \in \mathbb{C}$. To each real number $0<\varepsilon \ll 1$, we associate a Hermitian metric $h_{\varepsilon}$ on $L$ by

$$
h_{\varepsilon}:=\left\{\sum_{\lambda=0}^{r} \varepsilon^{2 \lambda} \sigma_{\lambda} \bar{\sigma}_{\lambda}\right\}^{-1}=\left\{\sum_{\lambda=0}^{r}\left(\varepsilon^{\lambda} \sigma_{\lambda}\right)\left(\varepsilon^{\lambda} \bar{\sigma}_{\lambda}\right)\right\}^{-1} .
$$


Let $\omega(\varepsilon)$ denote the Ricci form $R\left(h_{\varepsilon}\right):=\sqrt{-1} \partial \bar{\partial} \log h_{\varepsilon}$ for $\left(L, h_{\varepsilon}\right)$. We then have $\omega(\varepsilon) \in \mathcal{K}$. The infinitesimal action of $\mathcal{Y}_{j}$ on $h_{\varepsilon}$ is given by

$$
\begin{aligned}
\mathcal{Y}_{j} h_{\varepsilon} & =-h_{\varepsilon}^{2}\left\{\sum_{\lambda=0}^{r} \varepsilon^{2 \lambda}\left(\mathcal{Y}_{j} \sigma_{\lambda}\right) \bar{\sigma}_{\lambda}\right\} \\
& =-\varepsilon h_{\varepsilon}^{2}\left\{\sum_{\lambda=1}^{r} \sum_{\mu=0}^{\lambda-1} \varepsilon^{\lambda-\mu-1} b_{j, \lambda, \mu}\left(\varepsilon^{\mu} \sigma_{\mu}\right)\left(\varepsilon^{\lambda} \bar{\sigma}_{\lambda}\right)\right\} .
\end{aligned}
$$

For each $i \in\{1,2, \ldots, \ell\}$ and $j \in\{1,2, \ldots, m\}$, consider the functions $\xi_{i} \in$ $\tilde{\mathfrak{k}}_{\omega(\varepsilon)}$ and $\eta_{j} \in \tilde{\mathfrak{g}}_{\omega(\varepsilon)}$ such that $\operatorname{grad}_{\omega(\varepsilon)}^{\mathbb{C}} \xi_{i}=\mathcal{X}_{i}$ and $\operatorname{grad}_{\omega(\varepsilon)}^{\mathbb{C}} \eta_{j}=\mathcal{Y}_{j}$. Then $\xi_{i}$ is real-valued, where $\eta_{j}$ is possibly complex-valued. By $\operatorname{grad}_{\omega(\varepsilon)}^{\mathbb{C}} h_{\varepsilon}^{-1}\left(\mathcal{Y}_{j} h_{\varepsilon}\right)=$ $\sqrt{-1} \mathcal{Y}_{j}(\mathrm{cf} .[\mathrm{M} 3])$,

$$
\sqrt{-1} \eta_{j}=h_{\varepsilon}^{-1}\left(\mathcal{Y}_{j} h_{\varepsilon}\right)-\int_{M} h_{\varepsilon}^{-1}\left(\mathcal{Y}_{j} h_{\varepsilon}\right)\{\omega(\varepsilon)\}^{n} / A_{\kappa} .
$$

Put $v_{\lambda}:=\varepsilon^{\lambda} \sigma_{\lambda}$ and $C_{0}:=\sum_{j=1}^{m}\left\{\sum_{\lambda=1}^{r} \sum_{\mu=0}^{\lambda-1}\left|b_{j, \lambda, \mu}\right|^{2}\right\}^{1 / 2}$. Moreover, let $a_{j, \lambda, \mu}$ denote the complex number $\varepsilon^{\lambda-\mu-1} b_{j, \lambda, \mu}$ or 0 , according as $\lambda>\mu$ or $\lambda \leq \mu$. We then put $w_{j, \lambda}:=\sum_{\mu=0}^{r} a_{j, \lambda, \mu} v_{\mu}$. In view of (5.1), the CauchySchwarz inequality allows us to estimate the absolute value $\left|h_{\varepsilon}^{-1}\left(\mathcal{Y}_{j} h_{\varepsilon}\right)\right|$ of $h_{\varepsilon}^{-1}\left(\mathcal{Y}_{j} h_{\varepsilon}\right)$ as follows:

$$
\begin{aligned}
\left|h_{\varepsilon}^{-1}\left(\mathcal{Y}_{j} h_{\varepsilon}\right)\right|^{2} & =\varepsilon^{2} \frac{\left|\sum_{\lambda=1}^{r} w_{j, \lambda} \bar{v}_{\lambda}\right|^{2}}{\left(\sum_{\lambda=0}^{r} v_{\lambda} \bar{v}_{\lambda}\right)^{2}} \leq \varepsilon^{2} \frac{\left(\sum_{\lambda=1}^{r} w_{j, \lambda} \bar{w}_{j, \lambda}\right)\left(\sum_{\lambda=1}^{r} v_{\lambda} \bar{v}_{\lambda}\right)}{\left(\sum_{\lambda=0}^{r} v_{\lambda} \bar{v}_{\lambda}\right)^{2}} \\
& \leq \varepsilon^{2} \frac{\sum_{\lambda=1}^{r} w_{j, \lambda} \bar{w}_{j, \lambda}}{\sum_{\lambda=0}^{r} v_{\lambda} \bar{v}_{\lambda}} \leq \varepsilon^{2} \sum_{\lambda=1}^{r} \sum_{\mu=0}^{r}\left|a_{j, \lambda, \mu}\right|^{2} \leq\left(C_{0} \varepsilon\right)^{2} .
\end{aligned}
$$

This together with (5.2) implies $\left|\eta_{j}\right| \leq 2 C_{0} \varepsilon$ for all $j$. Now for $i \in$ $\{1,2, \ldots, \ell\}$ and $j, j^{\prime} \in\{1,2, \ldots, m\}$, the bilinear pairings $\left(\mathcal{X}_{i}, \mathcal{Y}_{j}\right)_{\kappa}$, $\left(\mathcal{Y}_{j}, \mathcal{Y}_{j^{\prime}}\right)_{\kappa}$ on $\mathfrak{g}$ (cf. [FM]; p.208) are estimated by

$$
\begin{aligned}
\left|\left(\mathcal{X}_{i}, \mathcal{Y}_{j}\right)_{\kappa}\right|^{2} & =\left|\int_{M} \xi_{i} \eta_{j}\{\omega(\varepsilon)\}^{n} / A_{\kappa}\right|^{2} \\
& \leq \int_{M} \xi_{i}^{2}\{\omega(\varepsilon)\}^{n} / A_{\kappa} \int_{M}\left|\eta_{j}\right|^{2}\{\omega(\varepsilon)\}^{n} / A_{\kappa} \\
& \leq\left(\mathcal{X}_{i}, \mathcal{X}_{i}\right)_{\kappa} \int_{M}\left|\eta_{j}\right|^{2}\{\omega(\varepsilon)\}^{n} / A_{\kappa} \leq 4 C_{0}^{2} \varepsilon^{2}\left(\mathcal{X}_{i}, \mathcal{X}_{i}\right)_{\kappa}, \\
\left|\left(\mathcal{Y}_{i}, \mathcal{Y}_{j^{\prime}}\right)_{\kappa}\right| & =\left|\int_{M} \eta_{j} \eta_{j^{\prime}}\{\omega(\varepsilon)\}^{n} / A_{\kappa}\right| \leq \int_{M}\left|\eta_{j} \eta_{j^{\prime}}\right|\{\omega(\varepsilon)\}^{n} / A_{\kappa} \leq 4 C_{0}^{2} \varepsilon^{2},
\end{aligned}
$$


where $\left(\mathcal{X}_{i}, \mathcal{X}_{i}\right)_{\kappa}=\int_{M} \xi_{i}^{2}\{\omega(\varepsilon)\}^{n} / A_{\kappa}>0$ is independent of the choice of $\varepsilon$ (cf. [FM;p.208]). By letting $\varepsilon \rightarrow 0$, we have $\left(\mathcal{X}_{i}, \mathcal{Y}_{j}\right)_{\kappa}=\left(\mathcal{Y}_{j}, \mathcal{Y}_{j^{\prime}}\right)_{\kappa}=0$. Hence,

TheOrem 5.3. $\mathfrak{u}=\left\{Z \in \mathfrak{g} ;(Z, W)_{\kappa}=0\right.$ for all $\left.W \in \mathfrak{g}\right\}$.

Remark. Let $\mathfrak{g}^{q+1} \ni\left(W_{0}, W_{1}, \ldots, W_{q}\right) \mapsto\left(W_{0}, W_{1}, W_{2}, \ldots, W_{q}\right)_{\kappa} \in \mathbb{C}$ be the symmetric $\mathbb{C}$-multilinear form as defined in [FM; p.209], where $q$ is an arbitrary positive integer. Then by the same argument as above, we can easily show that $\mathfrak{u}=\left\{Z \in \mathfrak{g} ;\left(Z, W_{1}, \ldots, W_{q}\right)_{\kappa}=0\right.$ for all $\left(W_{1}, \ldots, W_{q}\right) \in$ $\left.\mathfrak{g}^{q}\right\}$.

For each $\omega^{\prime} \in \mathcal{K}$, let $f_{\omega^{\prime}}$ denote the real-valued $C^{\infty}$ function on $M$ such that $\sigma\left(\omega^{\prime}\right)-C_{\kappa}=\square_{\omega^{\prime}} f_{\omega^{\prime}}$. The associated Futaki character $F_{\kappa}: \mathfrak{g} \rightarrow \mathbb{C}$ is defined by

$$
F_{\kappa}(W):=(\sqrt{-1})^{-1} \int_{M}\left(W f_{\omega^{\prime}}\right) \omega^{\prime n} / A_{\kappa}, \quad W \in \mathfrak{g} .
$$

This $F_{\kappa}$ depends only on $\kappa$ and is independent of the choice of $\omega^{\prime}$ in $\mathcal{K}$. Each element $W$ in $\mathfrak{g}$ is written as $\operatorname{grad}_{\omega^{\prime}}^{\mathbb{C}} \phi$ for some unique $\phi \in \tilde{\mathfrak{g}}_{\omega^{\prime}}$. Then

$$
F_{\kappa}(W)=\int_{M}\left(\sigma\left(\omega^{\prime}\right)-C_{\kappa}\right) \phi \omega^{\prime n} / A_{\kappa}
$$

in view of the computation in $[\mathrm{FM} ;(2.1)]$ (see also [LS]). We now consider a one-parameter subgroup $g_{t}:=\exp \left(t Z_{\mathbb{R}}\right), t \in \mathbb{R}$, of $G$, under the assumption that

$$
\omega^{\prime} \in \underline{\mathcal{K}} \quad \text { and } \quad Z \in \mathfrak{z}(V) .
$$

Since $\mathfrak{g}$ is a direct sum $\mathfrak{k}_{\omega^{\prime}}^{\mathbb{C}} \oplus \mathfrak{u}$ as a vector space, $Z$ is written as a sum $X+Y$ for some $X \in \mathfrak{k}_{\omega^{\prime}}^{\mathbb{C}}$ and $Y \in \mathfrak{u}$, where there uniquely exist $\xi \in \tilde{\mathfrak{k}}_{\omega^{\prime}}^{\mathbb{C}}$ and $\eta \in \tilde{\mathfrak{g}}_{\omega^{\prime}}$ such that $X=\operatorname{grad}_{\omega^{\prime}}^{\mathbb{C}} \xi$ and $Y=\operatorname{grad}_{\omega^{\prime}}^{\mathbb{C}} \eta$. Note also that $\omega_{t}:=g_{t}^{*} \omega^{\prime}$ is written uniquely as $\omega_{\psi_{t}}$ for some smooth path $\left\{\psi_{t} ; t \in \mathbb{R}\right\}$ in $\tilde{\mathcal{K}}$ satisfying $\int_{M} \dot{\psi}_{t} \omega_{t}^{n}=0$ for all $t \in \mathbb{R}$. Then $\dot{\psi}_{t}=2 \operatorname{Im}(\xi+\eta)$ at $t=0$. Since $\sigma\left(\omega^{\prime}\right)-\operatorname{pr}_{\omega^{\prime}} \sigma\left(\omega^{\prime}\right) \in\left(\mathbb{R} \oplus \tilde{\mathfrak{k}}_{\omega^{\prime}}\right)^{\perp}$, we obtain

$$
\begin{aligned}
& \left(\int_{M}\left\{\sigma\left(\omega_{t}\right)-\operatorname{pr}_{\omega_{t}} \sigma\left(\omega_{t}\right)\right\} \dot{\psi}_{t} \omega_{t}^{n} / A_{\kappa}\right)_{\mid t=0} \\
= & 2 \operatorname{Im}\left(\int_{M}\left\{\sigma\left(\omega^{\prime}\right)-\operatorname{pr}_{\omega^{\prime}} \sigma\left(\omega^{\prime}\right)\right\}(\xi+\eta) \omega^{\prime n} / A_{\kappa}\right) \\
= & 2 \operatorname{Im}\left(\int_{M}\left\{\sigma\left(\omega^{\prime}\right)-\operatorname{pr}_{\omega^{\prime}} \sigma\left(\omega^{\prime}\right)\right\} \eta \omega^{\prime n} / A_{\kappa}\right) .
\end{aligned}
$$


On the other hand, by Theorem 5.3, $\int_{M} \operatorname{pr}_{\omega^{\prime}} \sigma\left(\omega^{\prime}\right) \eta \omega^{\prime n} / A_{\kappa}=0$. Further by (5.4) and [N1], $\int_{M} \sigma\left(\omega^{\prime}\right) \eta \omega^{\prime n} / A_{\kappa}=\int_{M}\left(\sigma\left(\omega^{\prime}\right)-C_{\kappa}\right) \eta \omega^{\prime n} / A_{\kappa}=F_{\kappa}(\mathcal{Y})=0$. Hence,

$$
\left(\int_{M}\left\{\sigma\left(\omega_{t}\right)-\operatorname{pr}_{\omega_{t}} \sigma\left(\omega_{t}\right)\right\} \dot{\psi}_{t} \omega_{t}^{n} / A_{\kappa}\right)_{\mid t=0}=0 .
$$

Let $V$ and $\hat{\mu}_{V}: \mathcal{H}_{V} \rightarrow \mathbb{R}$ be as in the previous section. For each $g \in G$, the extremal Kähler vector field for $g^{*} \omega$ is $V^{g}:=\left(g^{-1}\right)_{*} V=\operatorname{Ad}\left(g^{-1}\right) V$. Replacing $V$ by $V^{g}$ in the definition of $\mathcal{H}_{V}$, we obtain

$$
\mathcal{H}_{V^{g}}:=\left\{\omega^{\prime} \in \underline{\mathcal{K}} ; \mathcal{V}_{\omega^{\prime}}=V^{g}\right\}
$$

which is just the pullback $g^{*} \mathcal{H}_{V}$ of $\mathcal{H}_{V}$ via $g$. Then the corresponding functional which replaces $\hat{\mu}_{V}$ will be denoted by $\hat{\mu}^{g}: \mathcal{H}_{V^{g}} \rightarrow \mathbb{R}$. We can actually define $\hat{\mu}^{g}: \mathcal{H}_{V^{g}} \rightarrow \mathbb{R}$ by

$$
\hat{\mu}^{g}\left(g^{*} \omega^{\prime}\right):=\hat{\mu}_{V}\left(\omega^{\prime}\right) \quad \text { for all } \omega^{\prime} \in \mathcal{H}_{V}
$$

where by $(3.1)$, the functionals $\hat{\mu}^{g}$ and $\hat{\mu}_{V^{g}}:=\mu+\eta_{V^{g}}$ on $\mathcal{H}_{V^{g}}$ differ just by a constant. Hence, if $V^{g_{1}}=V^{g_{2}}$ for some $g_{1}, g_{2} \in G$, the corresponding functionals $\hat{\mu}^{g_{1}}, \hat{\mu}^{g_{2}}$ differ by a constant. Obviously, $\hat{\mu}_{e}$ is just $\hat{\mu}_{V}$ if $e$ is the unit of $G$. Note that $\mathcal{H}_{V^{g_{1}}} \cap \mathcal{H}_{V^{g_{2}}}=\emptyset$ if $V^{g_{1}} \neq V^{g_{2}}$. In view of

$$
\underline{\mathcal{K}}=\bigcup_{g \in G} \mathcal{H}_{V^{g}}
$$

the functionals $\hat{\mu}^{g}: \mathcal{H}_{V^{g}} \rightarrow \mathbb{R}, g \in G$, glue together to define a $G$-invariant functional $\hat{\mu}: \underline{\mathcal{K}} \rightarrow \mathbb{R}$ on $\underline{\mathcal{K}}$ satisfying the equality

$$
\hat{\mu}_{\mid \mathcal{H}_{V^{g}}}=\hat{\mu}^{g}, \quad \text { for all } g \in G
$$

if we can show Proposition 5.7 below. Here, the $G$-invariance of $\hat{\mu}$ means that the equality $\hat{\mu}\left(g^{*} \omega^{\prime}\right)=\hat{\mu}\left(\omega^{\prime}\right)$ holds for all pairs $\left(g, \omega^{\prime}\right)$ in $G \times \underline{\mathcal{K}}$.

Proposition 5.7. If $g \in Z(V)$, then $\hat{\mu}^{g}=\hat{\mu}_{V}$.

Proof. If $g \in Z(V)$, then $V^{g}=V$, and hence $\mathcal{H}_{V^{g}}=\mathcal{H}_{V}$. Let $\theta$ be an arbitrary element of $\mathcal{H}_{V}$. It then suffices to show $\hat{\mu}_{V}\left(g^{*} \theta\right)=\hat{\mu}_{V}(\theta)$ for all $g \in Z(G)$. Take an arbitrary element $X$ in $\mathfrak{z}(V)$, and we put $h_{t}:=\exp \left(t X_{\mathbb{R}}\right)$ 
and $\omega_{t}:=\left(h_{t}\right)^{*} \theta$ for each $t \in \mathbb{R}$. Since $Z(V)$ is connected, the proof is reduced to showing the following infinitesimal equality:

$$
\frac{d}{d t} \hat{\mu}_{V}\left(\omega_{t}\right)_{\mid t=0}=0
$$

For some smooth path $\left\{\psi_{t} ; t \in \mathbb{R}\right\}$ in $\tilde{\mathcal{K}}$ satisfying $\int_{M} \dot{\psi}_{t} \omega_{t}^{n} / A_{\kappa}=0, t \in \mathbb{R}$, the Kähler form $\omega_{t}$ above is written as $\omega_{\psi_{t}}$ for each $t$. Moreover, we write $X$ as $\operatorname{grad}_{\omega_{t}}^{\mathbb{C}} \phi_{t}$ for some $\phi_{t} \in \tilde{\mathfrak{g}}_{\omega_{t}}$. Then by (4.4) and (5.6), we have the following identity as required:

$$
\frac{d}{d t} \hat{\mu}_{V}\left(\omega_{t}\right)_{\mid t=0}=-\left(\int_{M}\left\{\sigma\left(\omega_{t}\right)-\operatorname{pr}_{\omega_{t}} \sigma\left(\omega_{t}\right)\right\} \dot{\psi}_{t} \omega_{t}^{n} / A_{\kappa}\right)_{\mid t=0}=0 .
$$

For every quantized pair $(M, \kappa)$, we can thus define a $G$-invariant functional $\hat{\mu}: \underline{\mathcal{K}} \rightarrow \mathbb{R}$ as above. By [C1], all extremal Kähler metrics in the cohomology class $\kappa$ belong to $\underline{\mathcal{K}}$. On the other hand, the definition of $\hat{\mu}$ shows that

THEOREM 5.8. An element $\omega^{\prime}$ of $\underline{\mathcal{K}}$ is a critical point for the functional $\hat{\mu}: \underline{\mathcal{K}} \rightarrow \mathbb{R}$ if and only if $\omega^{\prime}$ is an extremal Kähler metric.

Remark. In this remark, we delete the assumption that the pair $(M, \kappa)$ is quantized. Suppose that the Kähler class $\kappa$ admits an extremal Kähler metric $\omega$. Let $V:=\mathcal{V}_{\omega}$ be the associated extremal Kähler vector field. Then by $[\mathrm{C} 1 ;(3.9)]^{3}$, the subgroups $Z(V)$ and $K_{\omega}^{\mathbb{C}}$ of $G$ coincide. Hence, in this case, the functionals $\hat{\mu}^{g}: \mathcal{H}_{V^{g}} \rightarrow \mathbb{R}, g \in G$, glue together to define a $G$ invariant functional $\hat{\mu}: \underline{\mathcal{K}} \rightarrow \mathbb{R}$ such that Theorem 5.8 above is valid even when the pair $(M, \kappa)$ is not necessarily quantized.

\section{$\S 6$. Functional $\hat{\nu}$ whose critical points are "Kähler-Einstein metrics"}

Throughout this section this section, we assume that the Kähler class $\kappa$ in the introduction is $2 \pi c_{1}(M)_{\mathbb{R}}$. Moreover, the anticanonical line bundle $K_{M}^{-1}$ of $M$ is chosen as the line bundle $L$ in $\S 5$. Since the $G$-action on $M$ naturally lifts to a $G$-action on $K_{M}$, the pair $(M, \kappa)$ is quantized in the

\footnotetext{
${ }^{3}$ In the decomposition $\mathfrak{h}=\mathfrak{a} \oplus \mathfrak{k}^{\prime} \oplus \mathfrak{m} \oplus \sum_{\lambda>0} \mathfrak{h}_{\lambda}=\mathfrak{a} \oplus \mathfrak{h}_{\mathcal{O}}^{\prime} \oplus \sum_{\lambda>0} \mathfrak{h}_{\lambda}$ in [C1; (3.9)], note that the vector spaces $\mathfrak{k}^{\prime} \oplus \mathfrak{m} \oplus \sum_{\lambda>0} \mathfrak{h}_{\lambda}, \mathfrak{h}_{0}^{\prime}, \mathfrak{k}^{\prime} \oplus \mathfrak{m}$ are respectively $\mathfrak{g}, \mathfrak{z}(V)$, $\mathfrak{k}_{\omega} \oplus \sqrt{-1} \mathfrak{k}_{\omega}=\mathfrak{k}_{\omega}^{\mathbb{C}}$ in our notation.
} 
sense of $\S 5$. By $\emptyset \neq \underline{\mathcal{K}} \subset \mathcal{K}$, we fix first of all an element $\omega$ in $\underline{\mathcal{K}}$. Then we have a unique element $\theta$ of $\underline{\mathcal{K}}$ such that $R(\theta)=\omega$. As in [BM1] (see also [BM2] ), we assign to each pair $\left(\theta^{\prime}, \theta^{\prime \prime}\right) \in \mathcal{K} \times \mathcal{K}$ a real number $N\left(\theta^{\prime}, \theta^{\prime \prime}\right) \in \mathbb{R}$ by

$$
N\left(\theta^{\prime}, \theta^{\prime \prime}\right):=\int_{a}^{b}\left\{\int_{M}\left(\square_{\theta_{t}} \dot{u}_{t}\right) R\left(\theta_{t}\right)^{n} / A_{\kappa}\right\} d t,
$$

where $\left\{u_{t} ; a \leq t \leq b\right\}$ is an arbitrary piecewise smooth path in $\tilde{\mathcal{K}}$ such that the associated path $\theta_{t}:=\omega_{u_{t}}, a \leq t \leq b$, in $\mathcal{K}$ satisfies $\theta^{\prime}=\theta_{a}$ and $\theta^{\prime \prime}=\theta_{b}$. Let $D_{\omega}: \tilde{\mathcal{K}} \rightarrow \mathbb{R}$ be the functional in [D1] (see also [DT]) defined by

$$
\begin{aligned}
D_{\omega}(\psi):= & \sqrt{-1} \sum_{i=0}^{n-1} \frac{n-i}{n+1} \int_{M} \partial \psi \wedge \bar{\partial} \psi \wedge \omega^{n-1-i} \wedge \omega_{\psi}^{i} / A_{\kappa} \\
& -\int_{M} \psi \omega^{n} / A_{\kappa}-\log \left(\int_{M} e^{f_{\omega}-\psi} \omega^{n} / A_{\kappa}\right),
\end{aligned}
$$

where for each $\omega^{\prime} \in \mathcal{K}$, the function $f_{\omega^{\prime}} \in C^{\infty}(M)_{\mathbb{R}}$ is defined by the equalities $R\left(\omega^{\prime}\right)=\omega^{\prime}+\sqrt{-1} \partial \bar{\partial} f_{\omega^{\prime}}$ and $\int_{M}\left(1-e^{f_{\omega^{\prime}}}\right) \omega^{\prime n} / A_{\kappa}=0$. For each $\psi \in \tilde{\mathcal{K}}$, let $\theta^{\psi}$ denote the unique element in $\mathcal{K}$ defined by $R\left(\theta^{\psi}\right)=\omega_{\psi}$. It is easy to check that

$$
D_{\omega}(\psi)=N\left(\theta, \theta^{\psi}\right) .
$$

Define a functional $\nu: \mathcal{K} \rightarrow \mathbb{R}$ by setting $\nu\left(\omega_{\psi}\right):=D_{\omega}(\psi)=N\left(\theta, \theta^{\psi}\right)$ for each $\psi \in \tilde{\mathcal{K}}$. Given a pair $\left(\omega^{\prime}, \omega^{\prime \prime}\right) \in \mathcal{K} \times \mathcal{K}$, let us consider an arbitrary smooth path $\left\{\psi_{t} ; a \leq t \leq b\right\}$ in $\tilde{\mathcal{K}}$ such that $\omega^{\prime}=\omega_{a}$ and $\omega^{\prime \prime}=\omega_{a}$, where we set $\omega_{t}:=\omega_{\psi_{t}}, a \leq t \leq b$, for simplicity. Then

$$
\frac{d}{d t} \nu\left(\omega_{t}\right)=-\int_{M}\left(1-e^{f_{\omega_{t}}}\right) \dot{\psi}_{t} \omega_{t}^{n} / A_{\kappa}, \quad a \leq t \leq b,
$$

and the set of the critical points for $\nu$ consists of all Kähler-Einstein metrics on $M$. Let $W \in \mathfrak{g}$ and $\omega^{\prime} \in \mathcal{K}$. Then $W=\operatorname{grad}_{\omega^{\prime}}^{\mathbb{C}} \phi$ for some $\phi \in \tilde{\mathfrak{g}}_{\omega^{\prime}}$. By the same computation as in $[\mathrm{M} 4 ; \S 2]$, we obtain

$$
\int_{M}\left(1-e^{f_{\omega^{\prime}}}\right) \phi \omega^{\prime n} / A_{\kappa}=\int_{M}\left(\sigma\left(\omega^{\prime}\right)-n\right) \phi \omega^{\prime n} / A_{\kappa}=F_{\kappa}(W),
$$

where for $\kappa$ as above, we have $C_{\kappa}=n$. As in the last section, let $V$ denote the extremal Kähler vector field $\mathcal{V}_{\omega}$ of $(M, \omega)$. Define a functional $\hat{\nu}_{V}: \mathcal{H}_{V} \rightarrow \mathbb{R}$ by

$$
\hat{\nu}_{V}:=\nu+\eta_{V}
$$


Then by [M4; 2.1], we see that $V=\operatorname{grad}_{\omega_{t}}^{\mathbb{C}} \operatorname{pr}_{\omega_{t}} \sigma\left(\omega_{t}\right)=\operatorname{grad}_{\omega_{t}}^{\mathbb{C}} \operatorname{pr}_{\omega_{t}}\left(1-e^{f_{\omega_{t}}}\right)$. It now follows from (1.1) and (6.1) that

$$
\frac{d}{d t} \hat{\nu}_{V}\left(\omega_{t}\right)=-\int_{M}\left\{\left(1-e^{f_{\omega_{t}}}\right)-\operatorname{pr}_{\omega_{t}}\left(1-e^{f_{\omega_{t}}}\right)\right\} \dot{\psi}_{t} \omega_{t}^{n} / A_{\kappa},
$$

for all $a \leq t \leq b$. Recall that an element $\omega^{\prime}$ of $\mathcal{K}$ is called a "Kähler-Einstein metric" if $1-e^{f_{\omega^{\prime}} \in \tilde{\mathfrak{k}}_{\omega^{\prime}}}$ (cf. [M4]). We now obtain

Proposition 6.4. An element $\omega^{\prime}$ of $\mathcal{H}_{V}$ is a critical point for the functional $\hat{\nu}_{V}: \mathcal{H}_{V} \rightarrow \mathbb{R}$ if and only if $\omega^{\prime}$ is a "Kähler-Einstein metric" in the sense of $[\mathrm{M} 4]$.

For each $g \in G$, the extremal Kähler vector field for $g^{*} \omega$ is $V^{g}:=$ $\left(g^{-1}\right)_{*} V=\operatorname{Ad}\left(g^{-1}\right) V$. Furthermore, $\mathcal{H}_{V^{g}}=\left\{\omega^{\prime} \in \underline{\mathcal{K}} ; \mathcal{V}_{\omega^{\prime}}=V^{g}\right\}=g^{*} \mathcal{H}_{V}$. In view of (3.1), we can define the corresponding functional $\hat{\nu}^{g}: \mathcal{H}_{V^{g}} \rightarrow \mathbb{R}$ by

$$
\hat{\nu}^{g}\left(g^{*} \omega^{\prime}\right):=\hat{\nu}_{V}\left(\omega^{\prime}\right) \quad \text { for all } \omega^{\prime} \in \mathcal{H}_{V}
$$

Then $\hat{\nu}^{g}$ depends smoothly on $g \in G$, where $\hat{\nu}^{g}$ coincides with $\hat{\nu}_{V}$ if $g$ is the unit $e$ of $G$. Moreover, if $V^{g_{1}} \neq V^{g_{2}}$, then $\mathcal{H}_{V^{g_{1}}} \cap \mathcal{H}_{V^{g_{2}}}=\emptyset$. In view of

$$
\underline{\mathcal{K}}=\bigcup_{g \in G} \mathcal{H}_{V^{g}}
$$

the functionals $\hat{\nu}^{g}: \mathcal{H}_{V^{g}} \rightarrow \mathbb{R}, g \in G$, glue together to define a $G$-invariant functional $\hat{\nu}: \underline{\mathcal{K}} \rightarrow \mathbb{R}$ on $\underline{\mathcal{K}}$ in such a way that

$$
\hat{\nu}_{\mid \mathcal{H}_{V^{g}}}=\hat{\nu}^{g}, \quad \text { for all } g \in G
$$

if we can show Proposition 6.5 below, where the $G$-invariance of $\hat{\nu}$ means that the equality $\hat{\nu}\left(g^{*} \omega^{\prime}\right)=\hat{\nu}\left(\omega^{\prime}\right)$ holds for all pairs $\left(g, \omega^{\prime}\right)$ in $G \times \underline{\mathcal{K}}$.

Proposition 6.5. If $g \in Z(V)$, then $\hat{\nu}^{g}=\hat{\nu}_{V}$.

Proof. Let $X \in \mathfrak{z}(V)$ and $\theta \in \mathcal{H}_{V}$. Put $\omega_{t}:=\left(\exp t X_{\mathbb{R}}\right)^{*} \theta, t \in \mathbb{R}$. As in the proof of Proposition 5.7, it suffices to show

$$
\frac{d}{d t} \hat{\nu}_{V}\left(\omega_{t}\right)_{\mid t=0}=0
$$

Here, $\omega_{t}$ is written as $\omega_{\psi_{t}}$ for some smooth path $\left\{\psi_{t} ; t \in \mathbb{R}\right\}$ in $\tilde{\mathcal{K}}$, where $\int_{M} \dot{\psi}_{t} \omega_{t}^{n} / A_{\kappa}=0$ for all $t$. Moreover, write $X$ as $\operatorname{grad}_{\omega_{t}}^{\mathbb{C}} \phi_{t}$ for some $\phi_{t} \in \tilde{\mathfrak{g}}_{\omega_{t}}$. 
By (6.2), the arguments deducing (5.6) is valid even when we replace $\sigma\left(\omega^{\prime}\right)$ and $\sigma\left(\omega_{t}\right)$ respectively by $1-e^{f_{\omega^{\prime}}}$ and $1-e^{f_{\omega_{t}}}$. Therefore,

$$
\left(\int_{M}\left\{\left(1-e^{f_{\omega_{t}}}\right)-\operatorname{pr}_{\omega_{t}}\left(1-e^{f_{\omega_{t}}}\right)\right\} \dot{\psi}_{t} \omega_{t}^{n} / A_{\kappa}\right)_{\mid t=0}=0 .
$$

Then by (6.3) and (6.6), we obtain the following required identity:

$$
\frac{d}{d t} \hat{\nu}\left(\omega_{t}\right)_{\mid t=0}=-\left(\int_{M}\left\{\left(1-e^{f_{\omega_{t}}}\right)-\operatorname{pr}_{\omega_{t}}\left(1-e^{f_{\omega_{t}}}\right)\right\} \dot{\psi}_{t} \omega_{t}^{n} / A_{\kappa}\right)_{\mid t=0}=0 .
$$

Recall that all "Kähler-Einstein metrics" in the cohomology class $\kappa$ belong to $\underline{\mathcal{K}}$ (cf. $[\mathrm{M} 4 ; \S 4])$. From the definition of the functional $\hat{\nu}$ above, we further obtain:

THEOREM 6.7. An element $\omega^{\prime}$ of $\underline{\mathcal{K}}$ is a critical point for the functional $\hat{\nu}: \underline{\mathcal{K}} \rightarrow \mathbb{R}$ if and only if $\omega^{\prime}$ is a "Kähler-Einstein metric" in the sense of [M4].

\section{$\S 7$. Convexity of $\hat{\nu}$ applied to the proof of Theorem $\mathbf{C}$}

For each maximal compact subgroup $K$ of $G$, let $\mathcal{K}^{K}$ and $\tilde{\mathcal{K}}^{K}$ denote the set of all $K$-invariant elements in $\mathcal{K}$ and $\tilde{\mathcal{K}}$, respectively (cf. $\S 3$ ). Then $\underline{\mathcal{K}}$ is written in the form

$$
\underline{\mathcal{K}}=\cup_{K} \mathcal{K}^{K},
$$

where the union is taken over all maximal compact subgroups $K$ of $G$. For such a $K$, we always have $\mathcal{K}^{K} \neq \emptyset$, and there exists an element $\omega$ of $\underline{\mathcal{K}}$ such that $K_{\omega}=K$. Let $\mathfrak{k}$ be the Lie subalgebra of $\mathfrak{g}$ corresponding to the Lie subgroup $K$ of $G$. Then

$$
\mathcal{K}^{K}=\left\{\omega_{\psi} ; \psi \in \tilde{\mathcal{K}}^{K}\right\}=\left\{\omega^{\prime} \in \mathcal{K} ; \mathfrak{k}_{\omega^{\prime}}=\mathfrak{k}\right\} \subset \mathcal{H}_{V} \subset \underline{\mathcal{K}},
$$

where $V:=\mathcal{V}_{\omega}$ is the extremal Kähler vector field of the Kähler manifold $(M, \omega)$. Note that, on $\mathcal{K}^{K}$, the functionals $\hat{\nu}$ and $\hat{\nu}_{V}$ coincide. We induce connections on $\mathcal{K}^{K}$ and $\tilde{\mathcal{K}}$ respectively from the connections (cf. [M2]) on $\mathcal{K}$ and $\tilde{\mathcal{K}}$. The purpose of this section is to show that the functional $\hat{\nu}$ is convex when restricted to $\mathcal{K}^{K}$. As an application of the convexity, we also show the uniqueness of "Kähler-Einstein metrics" (see [M4]) for toric Fano manifolds, modulo connected group actions, by the method as used by [G1] for extremal Kähler metrics. 
Fix an arbitrary element $\omega_{0}$ of $\mathcal{K}^{K}$. Let $\zeta$ be a $K$-invariant element in $C^{\infty}(M)_{\mathbb{R}}$ such that $\int_{M} \zeta \omega_{0}^{n} / A_{\kappa}=0$. For an $0<\varepsilon \ll 1$, choose a smooth path $\psi=\left\{\psi_{t} ;-\varepsilon \leq t \leq \varepsilon\right\}$ in $\tilde{\mathcal{K}}^{K}$ such that $\dot{\psi}_{t \mid t=0}=\zeta$ and that $\int_{M} \dot{\psi}_{t} \omega_{t}^{n} / A_{\kappa}=0$ for all $t$, where the associated path

$$
\omega_{t}:=\omega_{\psi_{t}}, \quad-\varepsilon \leq t \leq \varepsilon,
$$

in $\mathcal{K}^{K}$ passes through $\omega_{0}$ at $t=0$. We now consider the smooth oneparameter family $\dot{\psi}$ of $C^{\infty}$ functions on $M$ defined by

$$
\dot{\psi}:=\left\{\dot{\psi}_{t} ;-\varepsilon \leq t \leq \varepsilon\right\}
$$

Let us write $\omega_{t}=\sum_{\alpha, \beta}\left(g_{t}\right)_{\alpha \bar{\beta}} d z^{\alpha} \wedge d z^{\bar{\beta}}$ by using a system $\left(z^{1}, \ldots, z^{n}\right)$ of holomorphic local coordinates on $M$. To each smooth one-parameter family $\eta=\left\{\eta_{t} ;-\varepsilon \leq t \leq \varepsilon\right\}$ of $C^{\infty}$ functions on $M$, we put

$$
\left(\frac{D}{\partial t} \eta\right)_{t}=\dot{\eta}_{t}-\frac{1}{2} \sum_{\alpha, \beta}\left(g_{t}\right)^{\bar{\beta} \alpha}\left(\frac{\partial \dot{\psi}_{t}}{\partial z^{\alpha}} \frac{\partial \eta_{t}}{\partial z^{\bar{\beta}}}+\frac{\partial \dot{\psi} t}{\partial z^{\bar{\beta}}} \frac{\partial \eta_{t}}{\partial z^{\alpha}}\right), \quad-\varepsilon \leq t \leq \varepsilon .
$$

Then $\frac{D}{\partial t} \eta=\left\{\left(\frac{D}{\partial t} \eta\right)_{t} ;-\varepsilon \leq t \leq \varepsilon\right\}$ is the smooth one-parameter family of $C^{\infty}$ functions on $M$ obtained as the covariant derivative of $\eta$ along the path $\psi$ (cf. [M2]). In tangential directions of $\mathcal{K}^{K}$, the Hessian Hess $\hat{\nu}$ of $\hat{\nu}$ at $\omega_{0}$ is given by

$$
\begin{aligned}
& (\operatorname{Hess} \hat{\nu})_{\omega_{0}}(\zeta, \zeta)=\frac{d^{2}}{d t^{2}} \hat{\nu}_{V}\left(\omega_{t}\right)_{\mid t=0} \\
& \quad+\int_{M}\left\{\left(1-e^{f_{\omega_{0}}}\right)-\operatorname{pr}_{\omega_{0}}\left(1-e^{f_{\omega_{0}}}\right)\right\}\left(\frac{D}{\partial t} \dot{\psi}\right)_{t=0} \omega_{0}^{n} / A_{\kappa}
\end{aligned}
$$

For required convexity, it now suffices to show that $(\operatorname{Hess} \hat{\nu})_{\omega_{0}}(\zeta, \zeta)$ above is always nonnegative. For smooth one-parameter families $\xi=\left\{\xi_{t} ;-\varepsilon \leq t \leq\right.$ $\varepsilon\}, \eta=\left\{\eta_{t} ;-\varepsilon \leq t \leq \varepsilon\right\}$ of $C^{\infty}$ functions on $M$, we define

$$
\langle\langle\xi, \eta\rangle\rangle_{t}=\int_{M} \xi_{t} \eta_{t} \omega_{t}^{n}
$$

where $\omega_{t},-\varepsilon \leq t \leq \varepsilon$, are as in (7.1). For the extremal Kähler vector field $V$, there exists a one-parameter family $\phi=\left\{\phi_{t} ;-\varepsilon \leq t \leq \varepsilon\right\}$ of real-valued $C^{\infty}$ functions on $M$ such that $\int_{M} \phi_{t} \omega_{t}^{n} / A_{\kappa}=0$ for all $t$, and that

$$
V=\operatorname{grad}_{\omega_{t}}^{\mathbb{C}} \phi_{t}, \quad-\varepsilon \leq t \leq \varepsilon
$$


Then by [FM],we have $\phi_{t}=\phi_{0}+\sqrt{-1} V \psi_{t}$. Since $\psi_{t}$ is a $K$-invariant function, and since $V \in \mathfrak{k}$, it follows that $(V+\bar{V}) \dot{\psi}_{t}=V_{\mathbb{R}} \dot{\psi}_{t}=0$, and therefore

$$
\begin{aligned}
\dot{\phi}_{t} & =\sqrt{-1} V \dot{\psi}_{t} \\
& =\frac{1}{2} \sum_{\alpha, \beta}\left(g_{t}\right)^{\bar{\beta} \alpha}\left(\frac{\partial \dot{\psi}_{t}}{\partial z^{\alpha}} \frac{\partial \phi_{t}}{\partial z^{\bar{\beta}}}+\frac{\partial \dot{\psi}_{t}}{\partial z^{\bar{\beta}}} \frac{\partial \phi_{t}}{\partial z^{\alpha}}\right), \text { i.e., } \frac{D}{\partial t} \phi=0,
\end{aligned}
$$

(see [G1]). On the other hand, by $\omega_{t} \in \mathcal{K}^{K}$, we have $V=\operatorname{grad}_{\omega_{t}}^{\mathbb{C}} \operatorname{pr}_{\omega_{t}}(1-$ $\left.e^{f_{\omega_{t}}}\right)$. It is now easy to check that $\phi_{t}=\operatorname{pr}_{\omega_{t}}\left(1-e^{f_{\omega_{t}}}\right)$ for all $t$. For simplicity, let $1-e^{f}$ denote the one-parameter family $\left\{1-e^{f_{\omega_{t}}} ;-\varepsilon \leq t \leq \varepsilon\right\}$ of $C^{\infty}$ functions on $M$. Then by (6.3),

$$
\frac{d}{d t} \hat{\nu}_{V}\left(\omega_{t}\right)=-\left\langle\left\langle 1-e^{f}-\phi, \dot{\psi}\right\rangle_{t} .\right.
$$

We now put $\varphi_{t}:=\psi_{t}+C_{t}$, where each $C_{t} \in \mathbb{R}$ is a constant depending smoothly on $t$ such that $\int_{M} \dot{\varphi}_{t} \widetilde{\omega_{t}^{n}}=0$ for all $t$. Here, $\widetilde{\omega_{t}^{n}}:=e^{f_{\omega_{t}}} \omega_{t}^{n} / A_{\kappa}$. We also let

$$
\tilde{\square}_{t}:=\square_{\omega_{t}}+\sum_{\alpha, \beta}\left(g_{t}\right)^{\bar{\beta} \alpha} \frac{\partial f_{\omega_{t}}}{\partial z^{\alpha}} \frac{\partial}{\partial z^{\bar{\beta}}} .
$$

Consider the smooth one-parameter family $\dot{\varphi}:=\left\{\dot{\varphi}_{t} ;-\varepsilon \leq t \leq \varepsilon\right\}$ of $C^{\infty}$ functions on $M$. Then by $\left\langle\left\langle 1-e^{f}-\phi, \dot{\psi}\right\rangle\right\rangle_{t}=\left\langle\left\langle 1-e^{f}-\phi, \dot{\varphi}\right\rangle\right\rangle_{t}$, replacing $\dot{\psi}$ by $\dot{\varphi}$ in (7.4) and differentiating this with respect to $t$, we obtain

$$
\frac{d^{2}}{d t^{2}} \hat{\nu}_{V}\left(\omega_{t}\right)=-\left\langle\left\langle 1-e^{f}-\phi, \frac{D}{\partial t} \dot{\varphi}\right\rangle\right\rangle_{t}-\left\langle\left\langle\frac{D}{\partial t}\left(1-e^{f}-\phi\right), \dot{\varphi}\right\rangle\right\rangle_{t} .
$$

Therefore, it follows from (7.2), (7.3) and (7.5) that

$$
\begin{aligned}
& (\operatorname{Hess} \hat{\nu})_{\omega_{0}}(\zeta, \zeta)=\frac{d^{2}}{d t^{2}} \hat{\nu}_{V}\left(\omega_{t}\right)_{\mid t=0}+\left\langle\left\langle 1-e^{f}-\phi, \frac{D}{\partial t} \dot{\psi}\right\rangle\right\rangle_{t=0} \\
= & \frac{d^{2}}{d t^{2}} \hat{\nu}_{V}\left(\omega_{t}\right)_{\mid t=0}+\left\langle\left\langle 1-e^{f}-\phi, \frac{D}{\partial t} \dot{\varphi}\right\rangle\right\rangle_{t=0} \\
= & -\left\langle\left\langle\frac{D}{\partial t}\left(1-e^{f}-\phi\right), \dot{\varphi}\right\rangle\right\rangle_{t=0}=-\left\langle\left\langle\frac{D}{\partial t}\left(1-e^{f}\right), \dot{\varphi}\right\rangle\right\rangle_{t=0} .
\end{aligned}
$$

For simplicity, we put $f_{t}:=f_{\omega_{t}}$. Recall that $\dot{f}_{t}=-\square_{\omega_{t}} \dot{\varphi}_{t}-\dot{\varphi}_{t}+B_{t}$ for some constant $B_{t} \in \mathbb{R}$ (cf. [F1]). Let $\operatorname{Re}(\ldots)$ denote the real part. Then by

$$
-\left\langle\left\langle\frac{D}{\partial t}\left(1-e^{f}\right), \dot{\varphi}\right\rangle\right\rangle_{t}
$$




$$
=\int_{M}\left\{\dot{f}_{t}-\frac{1}{2} \sum_{\alpha, \beta}\left(g_{t}\right)^{\bar{\beta} \alpha}\left(\frac{\partial \dot{\psi}_{t}}{\partial z^{\alpha}} \frac{\partial f_{t}}{\partial z^{\bar{\beta}}}+\frac{\partial \dot{\psi_{t}}}{\partial z^{\bar{\beta}}} \frac{\partial f_{t}}{\partial z^{\alpha}}\right)\right\} \dot{\dot{\varphi}_{t}} \widetilde{\omega_{t}^{n}}
$$

and by $\int_{M} \dot{\varphi}_{t} \widetilde{\omega_{t}^{n}}=0$, we now obtain

$$
\begin{aligned}
-\left\langle\left\langle\frac{D}{\partial t}\left(1-e^{f}\right), \dot{\varphi}\right\rangle\right\rangle_{t} & =\int_{M}\left\{-\operatorname{Re}\left(\tilde{\square}_{t} \dot{\varphi}_{t}\right)-\dot{\varphi}_{t}\right\} \dot{\varphi}_{t} \widetilde{\omega_{t}^{n}} \\
& =\operatorname{Re}\left\{\int_{M}-\left(\tilde{\square}_{t} \dot{\varphi}_{t}+\dot{\varphi}_{t}\right) \dot{\varphi}_{t} \widetilde{\omega_{t}^{n}}\right\} \geq 0,
\end{aligned}
$$

since the eigenvalues of $-\tilde{\square}_{t}$ are all real, and its first positive eigenvalue is bouded from below by 1 (cf. [F2]). Thus (Hess $\hat{\nu})_{\omega_{0}}(\zeta, \zeta) \geq 0$, as required.

Remark. Let $M$ be a nonsingular toric variety defined over $\mathbb{C}$. By the convexity of $\hat{\mu}_{V}$ along $\mathcal{K}^{K}$, [G1] shows that the extremal Kähler metrics in each Kähler class are unique up to the action of $G=\operatorname{Aut}^{0}(M)$. By the convexity of $\hat{\nu}$ along $\mathcal{K}^{K}$ shown just above, we can similarly prove in (7.6) the uniqueness of "Kähler-Einstein metrics" up to the action of $G=\operatorname{Aut}^{0}(M)$ when $M$ is a nonsingular toric Fano variety.

(7.6) Proof of Theorem C. Let $\mathcal{E}$ be the set of all "Kähler-Einstein metrics" (cf. [M4]) in the class $2 \pi c_{1}(M)_{\mathbb{R}}$. It then suffices to show that $\mathcal{E}$ is connected. Let $\omega_{0}, \omega_{1} \in \mathcal{E}$. Replacing $\omega_{1}$ by $g^{*} \omega_{1}$ for some $g \in G$ if necessary, we may assume that both $\omega_{0}$ and $\omega_{1}$ belong to $\mathcal{K}^{K}$ for some maximal compact subgroup $K$ of $G$. Since $M$ is toric, the arguments as in [G1] allows us to connect $\omega_{0}$ and $\omega_{1}$ by a geodesic $\omega_{t}, 0 \leq t \leq 1$, in $\mathcal{K}^{K}$. In view of the convexity of $\hat{\nu}$ along $\mathcal{K}^{K}$, we have

$$
\begin{aligned}
& \frac{d}{d t} \hat{\nu}\left(\omega_{t}\right)_{\mid t=0}=\frac{d}{d t} \hat{\nu}\left(\omega_{t}\right)_{\mid t=1}=0 ; \\
& \frac{d^{2}}{d t^{2}} \hat{\nu}\left(\omega_{t}\right) \geq 0, \quad 0 \leq t \leq 1 .
\end{aligned}
$$

Therefore, $\hat{\nu}\left(\omega_{t}\right)$ is constant on the closed interval $\{0 \leq t \leq 1\}$. Then it is easily seen that $\hat{\nu}\left(\omega_{t}\right)$ is a critical point of $\hat{\nu}$ for all $t$, and hence $\mathcal{E}$ is connected. (In fact, the geodesic $\omega_{t}, 0 \leq t \leq 1$, can be written as $^{4}$

$$
\omega_{t}=\left\{\exp \left(t Z_{\mathbb{R}}\right)\right\}^{*} \omega_{0}
$$

\footnotetext{
${ }^{4}$ In relation to this expression, we here note that Theorem 3.5 in [M2] is true under the additional assumption that $Y$ is in the center of $\mathfrak{k}_{\omega}^{\mathbb{C}}$, though it is incorrect without any such assumption.
} 
for some $Z \in \sqrt{-1} \mathfrak{z}(\mathfrak{k})$, where $\mathfrak{z}(\mathfrak{k})$ denotes the center of $\mathfrak{k}$. )

\section{REFERENCES}

[BM1] S. Bando and T. Mabuchi, On some integral invariants on complex manifolds I, Proc. Japan Acad., 62 (1986), 197-200.

[BM2] Uniqueness of Einstein Kähler metrics modulo connected group actions, Algebraic Geometry, Sendai, 1985, Adv. Stud. Pure Math. 10, Kinokuniya and North-Holland, Tokyo and Amsterdam (1987), pp. 11-40.

[C1] E. Calabi, Extremal Kähler metrics II, Differential geometry and complex analysis (I. Chavel and H.M. Farkas, eds.), Springer-Verlag, Heidelberg (1985), pp. 95-114.

[D1] W. Ding, Remarks on the existence problem of positive Kähler-Einstein metrics, Math. Ann., 282 (1988), 463-471.

[DT] W. Ding and G. Tian, The generalized Moser-Truginger inequality, Proc. Nankai Internat. Conf. Nonlinear Analysis (1993).

[Fj] A. Fujiki, On automorphism groups of compact Kähler manifolds, Invent. Math., 44 (1978), 225-258.

[F1] A. Futaki, An obstruction to the existence of Einstein Kähler metrics, Invent. Math., 73 (1983), 437-443.

[F2] A. Futaki, Kähler-Einstein metrics and integral invariants, Lect. Notes in Math. 1314, Springer-Verlag, Heidelberg, 1988.

[FM] A. Futaki and T. Mabuchi, Bilinear forms and extremal Kähler vector fields associated with Kähler classes, Math. Ann., 301 (1995), 199-210.

[G1] Z.D. Guan, On modified Mabuchi functional and Mabuchi moduli space of Kähler metrics on toric bundles, Math. Res. Letters, 6 (1999), 547-555.

[GC] Z.D. Guan and X. Chen, Existence of extremal metrics on almost homogeneous manifolds of cohomogeneity one, to appear in Asian J. Math..

[LS] C. LeBrun and S. Simanca, Extremal Kähler metrics and complex deformation theory, Geom. Funct. Anal., 4 (1994), 298-336.

[M1] T. Mabuchi, K-energy maps integrating Futaki invariants, Tôhoku Math. J., 38 (1986), 575-593.

[M2] T. Mabuchi, Some symplectic geometry on compact Kähler manifolds (I), Osaka J. Math., 24 (1987), 227-252.

[M3] T. Mabuchi, An algebraic character associated with Poisson brackets, Recent topics in differential and analytic geometry, Adv. Stud. Pure Math. 18-I, Kinokuniya and Academic Press, Tokyo and New York (1990), pp. 339-358.

[M4] T. Mabuchi, Kähler-Einstein metrics for manifolds with nonvanishing Futaki character, to appear in Tôhoku Math. J., 53 (2001).

[M5] T. Mabuchi, Multiplier Hermitian structures on Kähler manifolds, preprint.

[N1] Y. Nakagawa, Bando-Calabi-Futaki characters of Kähler orbifolds, Math. Ann., 314 (1999), 369-380.

[S1] S. Simanca, A K-energy characterization of extremal Kähler metrics, Proc. Amer. Math. Soc., 128 (2000), 1531-1535. 
Department of Mathematics, Osaka University

Toyonaka, Osaka, 560-0043

Japan

mabuchi@math.wani.osaka-u.ac.jp 